\title{
Extremal theory of locally sparse multigraphs
}

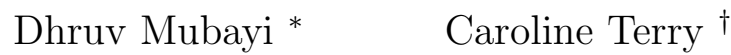

September 28, 2018

\begin{abstract}
An $(n, s, q)$-graph is an $n$-vertex multigraph where every set of $s$ vertices spans at most $q$ edges. In this paper, we determine the maximum product of the edge multiplicities in $(n, s, q)$ graphs if the congruence class of $q$ modulo $\left(\begin{array}{c}s \\ 2\end{array}\right)$ is in a certain interval of length about $3 s / 2$. The smallest case that falls outside this range is $(s, q)=(4,15)$, and here the answer is $a^{n^{2}+o\left(n^{2}\right)}$ where $a$ is transcendental assuming Schanuel's conjecture. This could indicate the difficulty of solving the problem in full generality. Many of our results can be seen as extending work by Bondy-Tuza [2] and Füredi-Kündgen [8] about sums of edge multiplicities to the product setting.

We also prove a variety of other extremal results for $(n, s, q)$-graphs, including productstability theorems. These results are of additional interest because they can be used to enumerate and to prove logical 0-1 laws for $(n, s, q)$-graphs. Our work therefore extends many classical enumerative results in extremal graph theory beginning with the Erdős-Kleitman-Rothschild theorem [6] to multigraphs.
\end{abstract}

\section{Introduction}

Given a set $X$ and a positive integer $t$, let $\left(\begin{array}{c}X \\ t\end{array}\right)=\{Y \subseteq X:|Y|=t\}$. A multigraph is a pair $(V, w)$, where $V$ is a set of vertices and $w:\left(\begin{array}{c}V \\ 2\end{array}\right) \rightarrow \mathbb{N}=\{0,1,2, \ldots\}$.

Definition 1. Given integers $s \geq 2$ and $q \geq 0$, a multigraph $(V, w)$ is an $(s, q)$-graph if for every $X \in\left(\begin{array}{l}V \\ s\end{array}\right)$ we have $\sum_{x y \in\left(\begin{array}{l}X \\ 2\end{array}\right)} w(x y) \leq q$. An $(n, s, q)$-graph is an $(s, q)$-graph with $n$ vertices, and $F(n, s, q)$ is the set of $(n, s, q)$-graphs with vertex set $[n]:=\{1, \ldots, n\}$.

The goal of this paper is to investigate extremal, structural, and enumeration problems for $(n, s, q)$-graphs for a large class of pairs $(s, q)$.

Definition 2. Given a multigraph $G=(V, w)$, define

$$
\begin{gathered}
S(G)=\sum_{x y \in\left(\begin{array}{c}
V \\
2
\end{array}\right)} w(x y) \quad \text { and } \quad P(G)=\prod_{x y \in\left(\begin{array}{c}
V \\
2
\end{array}\right)} w(x y), \\
\operatorname{ex}_{\Sigma}(n, s, q)=\max \{S(G): G \in F(n, s, q)\} \quad \text { and } \quad \operatorname{ex}_{\Pi}(n, s, q)=\max \{P(G): G \in F(n, s, q)\} .
\end{gathered}
$$

An $(n, s, q)$-graph $G$ is sum-extremal (product-extremal) if $S(G)=\operatorname{ex}_{\Sigma}(n, s, q)\left(P(G)=\operatorname{ex}_{\Pi}(n, s, q)\right.$ ). Let $\mathcal{S}(n, s, q)(\mathcal{P}(n, s, q))$ be the set of all sum-extremal (product-extremal) $(n, s, q)$-graphs with vertex set $[n]$.

\footnotetext{
${ }^{*}$ Department of Mathematics, Statistics, and Computer Science, University of Illinois at Chicago. Research supported in part by NSF Grant DMS 1300138; mubayi@uic.edu

${ }^{\dagger}$ Department of Mathematics, University of Maryland, College Park; cterry@umd.edu
} 
In [2], Bondy and Tuza determine the structure of multigraphs in $\mathcal{S}(n, s, q)$ when $n$ is large compared to $s$ and $q \equiv 0,-1\left(\bmod \left(\begin{array}{l}s \\ 2\end{array}\right)\right)$ and when $s=3$. In [9], Füredi and Kündgen (among other things) determine the asymptotic value of $\operatorname{ex}_{\Sigma}(n, s, q)$ for all $s, q$ with a $O(n)$ error term, and the exact value is determined for many cases. Other special cases of these questions have appeared in [13]. A natural next step from the investigation of extremal problems for $(n, s, q)$-graphs is to consider questions of structure and enumeration. The question of enumeration for $(n, s, q)$-graphs was first addressed in [14], where it was shown the problem is closely related extremal results for the product of the edge multiplicities.

Definition 3. Given integers $s \geq 2$ and $q \geq\left(\begin{array}{l}s \\ 2\end{array}\right)$, define the asymptotic product density and the asymptotic sum density, respectively, as the following limits (which both exist):

$$
\operatorname{ex}_{\Pi}(s, q)=\lim _{n \rightarrow \infty}\left(\operatorname{ex}_{\Pi}(n, s, q)\right)^{\frac{1}{\left(\begin{array}{l}
n \\
2
\end{array}\right)}} \quad \text { and } \quad \operatorname{ex}_{\Sigma}(s, q)=\lim _{n \rightarrow \infty} \frac{\operatorname{ex}_{\Sigma}(n, s, q)}{\left(\begin{array}{l}
n \\
2
\end{array}\right)} .
$$

In [14], the current authors showed $\operatorname{ex}_{\Pi}(s, q)$ exists for all $s \geq 2$ and $q \geq 0$ and proved the following enumeration theorem for $(n, s, q)$-graphs in terms of $\operatorname{ex}_{\Pi}\left(s, q+\left(\begin{array}{l}s \\ 2\end{array}\right)\right)$.

Theorem 1. ( [14]) Suppose $s \geq 2$ and $q \geq 0$ are integers. If $\operatorname{ex}_{\Pi}\left(s, q+\left(\begin{array}{l}s \\ 2\end{array}\right)\right)>1$, then

$$
\operatorname{ex}_{\Pi}\left(s, q+\left(\begin{array}{l}
s \\
2
\end{array}\right)\right)^{\left(\begin{array}{l}
n \\
2
\end{array}\right)} \leq|F(n, s, q)| \leq \operatorname{ex}_{\Pi}\left(s, q+\left(\begin{array}{l}
s \\
2
\end{array}\right)\right)^{(1+o(1))\left(\begin{array}{l}
n \\
2
\end{array}\right)},
$$

and if $\operatorname{ex}_{\Pi}\left(s, q+\left(\begin{array}{l}s \\ 2\end{array}\right)\right) \leq 1$, then $|F(n, s, q)| \leq 2^{o\left(n^{2}\right)}$.

This result was used in [14] along with a computation of $\operatorname{ex}_{\Pi}(4,15)$ to give an enumeration of $F(n, 4,9)$. This case was of particular interest because it turned out that $|F(n, 4,9)|=a^{n^{2}+o\left(n^{2}\right)}$, where $a$ is transcendental under the assumption of Schanuel's conjecture. In this paper, we continue this line of investigations by proving enumeration results for further cases of $s$ and $q$, and in some cases proving approximate structure theorems (the particular special case $(s, q)=(3,4)$ was recently studied in [7]). This generalizes many classical theorems about enumeration in extremal graph theory (beginning with the Erdős-Kleitman-Rothschild theorem [6]) to the multigraph setting. All of these results rely on computing $\operatorname{ex}_{\Pi}(n, s, q)$, characterizing the elements in $\mathcal{P}(n, s, q)$, and proving corresponding product-stability theorems, and this is the main content of this paper. Questions about $\operatorname{ex}_{\Pi}(n, s, q)$ and $\mathcal{P}(n, s, q)$ may also be of independent interest, as they are natural "product versions" of the questions about extremal sums for $(n, s, q)$-graphs investigated in [2, 9 .

\section{Main Results}

Given a multigraph $G=(V, w)$ and $x y \in\left(\begin{array}{c}V \\ 2\end{array}\right)$, we will refer to $w(x y)$ as the multiplicity of $x y$. The multiplicty of $G$ is $\mu(G)=\max \left\{w(x y): x y \in\left(\begin{array}{c}V \\ 2\end{array}\right)\right\}$. Our first main result, Theorem 2 below, gives us information about the asymptotic properties of elements in $F(n, s, q)$, in the case when $\operatorname{ex}_{\Pi}\left(s, q+\left(\begin{array}{l}s \\ 2\end{array}\right)\right)>1$. Suppose $G=(V, w)$ and $G^{\prime}=\left(V, w^{\prime}\right)$ are multigraphs. We say that $G$ is a submultigraph of $G^{\prime}$ if $V=V^{\prime}$ and for each $x y \in\left(\begin{array}{c}V \\ 2\end{array}\right), w(x y) \leq w^{\prime}(x y)$. Define $G^{+}=\left(V, w^{+}\right)$where for each $x y \in\left(\begin{array}{c}V \\ 2\end{array}\right), w^{+}(x y)=w(x y)+1$. Observe that if $G \in F(n, s, q)$, then $G^{+} \in F\left(n, s, q+\left(\begin{array}{l}s \\ 2\end{array}\right)\right)$.

Definition 4. Suppose $\epsilon>0$ and $n, s, q$ are integers satisfying $n \geq 1, s \geq 2$, and $q \geq 0$. Set

$$
\mathbb{E}(n, s, q, \epsilon)=\left\{G \in F(n, s, q): P\left(G^{+}\right)>\operatorname{ex}_{\Pi}\left(s, q+\left(\begin{array}{l}
s \\
2
\end{array}\right)\right)^{(1-\epsilon)\left(\begin{array}{l}
n \\
2
\end{array}\right)}\right\} .
$$

Then set $E(n, s, q, \epsilon)=\left\{G \in F(n, s, q): G\right.$ is a submultigraph of some $\left.G^{\prime} \in \mathbb{E}(n, s, q, \epsilon)\right\}$. 
Theorem 2. Suppose $s \geq 2$ and $q \geq 0$ are integers satisfying $\operatorname{ex}_{\Pi}\left(s, q+\left(\begin{array}{l}s \\ 2\end{array}\right)\right)>1$. Then for all $\epsilon>0$, there is $\beta>0$ such that for all sufficiently large $n$, the following holds.

$$
\frac{|F(n, s, q) \backslash E(n, s, q, \epsilon)|}{|F(n, s, q)|} \leq 2^{-\beta n^{2}} .
$$

Theorem 2 will be proved in Section 4 using a consequence of a version of the hypergraph containers theorem for multigraphs from [14]. Our next results investigate $\operatorname{ex}_{\Pi}(n, s, q)$ and $\mathcal{P}(n, s, q)$ for various values of $(s, q)$. Observe that if $q<\left(\begin{array}{c}s \\ 2\end{array}\right)$, then for any $n \geq s$, every $(n, s, q)$-graph $G$ must contain an edge of multiplicity 0 , and therefore $P(G)=0$. Consequently, $\operatorname{ex}_{\Pi}(n, s, q)=0$ and $\mathcal{P}(n, s, q)=F(n, s, q)$, for all $n \geq s$. For this reason we restrict our attention to the cases where $s \geq 2$ and $q \geq\left(\begin{array}{l}s \\ 2\end{array}\right)$. Suppose $G=(V, w)$ and $G^{\prime}=\left(V^{\prime}, w^{\prime}\right)$. Then $G=(V, w)$ and $G^{\prime}=\left(V^{\prime}, w^{\prime}\right)$ are isomorphic, denoted $G \cong G^{\prime}$, if there is a bijection $f: V \rightarrow V^{\prime}$ such that for all $x y \in\left(\begin{array}{c}V \\ 2\end{array}\right)$, $w(x y)=w^{\prime}(f(x) f(y))$. If $V=V^{\prime}$, set $\Delta\left(G, G^{\prime}\right)=\left\{x y \in\left(\begin{array}{c}V \\ 2\end{array}\right): w(x y) \neq w^{\prime}(x y)\right\}$. Given $\delta>0$, $G$ and $G^{\prime}$ are $\delta$-close if $\left|\Delta\left(G, G^{\prime}\right)\right| \leq \delta n^{2}$, otherwise they are $\delta$-far. If $X \subseteq V, G[X]$ denotes the multigraph $\left(X, w \Gamma_{\left(\begin{array}{c}X \\ 2\end{array}\right)}\right)$. Suppose that $q \equiv b\left(\bmod \left(\begin{array}{l}s \\ 2\end{array}\right)\right)$. Our results fall into three cases depending on the value of $b$.

\subsection{The case $0 \leq b \leq s-2$}

Definition 5. Given $n \geq s \geq 1$ and $a \geq 1$, let $\mathbb{U}_{s, a}(n)$ be the set of multigraphs $G=([n], w)$ such that there is a partition $A_{0}, A_{1}, \ldots, A_{\left\lfloor\frac{n}{s}\right\rfloor}$ of $[n]$ for which the following holds.

- For each $1 \leq i \leq\lfloor n / s\rfloor,\left|A_{i}\right|=s$, and $\left|A_{0}\right|=n-s\lfloor n / s\rfloor$.

- For each $0 \leq i \leq\lfloor n / s\rfloor$, and $G\left[A_{i}\right]$ is a star with $\left|A_{i}\right|-1$ edges of multiplicity $a+1$ and all other edges of multiplicity $a$.

- For all $x y \notin \bigcup\left(\begin{array}{c}A_{i} \\ 2\end{array}\right), w(x y)=a$.

Let $\mathbb{U}_{a}(n)$ be the unique element of $\mathbb{U}_{1, a}(n)$, i.e. $\mathbb{U}_{a}(n)=([n], w)$ where $w(x y)=a$ for all $x y \in\left(\begin{array}{c}{[n]} \\ 2\end{array}\right)$.

Theorem 3. Suppose $n, s, q, a$ are integers satisfying $n \geq s \geq 2, a \geq 1$, and $q=a\left(\begin{array}{l}s \\ 2\end{array}\right)+b$ for some $0 \leq b \leq s-2$.

- (Extremal) Then $a^{\left(\begin{array}{c}n \\ 2\end{array}\right)} \leq \operatorname{ex}_{\Pi}(n, s, q) \leq a^{\left(\begin{array}{c}n \\ 2\end{array}\right)}((a+1) / a)^{\left\lfloor\frac{b}{b+1} n\right\rfloor}$ and thus $\operatorname{ex}_{\Pi}(s, q)=a$. Further,

(a) If $b=0$, then $\mathcal{P}(n, s, q)=\left\{\mathbb{U}_{a}(n)\right\}$ and $\operatorname{ex}_{\Pi}(n, s, q)=a^{\left(\begin{array}{c}n \\ 2\end{array}\right)}$. (b) If $b=s-2$, then $\mathbb{U}_{s-1, a}(n) \subseteq \mathcal{P}(n, s, q)$ and $\operatorname{ex}_{\Pi}(n, s, q)=a^{\left(\begin{array}{c}n \\ 2\end{array}\right)\left(\frac{a+1}{a}\right)^{\left\lfloor\frac{(s-2) n}{s-1}\right\rfloor}}$. Also,
$\mathcal{P}(n, 3, q)=\mathbb{U}_{2, a}(n)$.

- (Stability) For all $\delta>0$, there is $\epsilon>0$ and $M$ such that for all $n>M$ and $G \in F(n, s, q)$, if $P(G)>\operatorname{ex}_{\Pi}(n, s, q)^{1-\epsilon}$, then $G$ is $\delta$-close to $\mathbb{U}_{a}(n)$.

One interesting phenomenon discovered in [2] is that $\mathcal{S}(n, 3,3 a+1)$ has many non-isomorphic multigraphs when $a \geq 1$ and $n$ is large. In contrast to this, Theorem 3 shows that all the multigraphs in $\mathcal{P}(n, 3,3 a+1)=\mathbb{U}_{2, a}(n)$ are isomorphic. 


\subsection{The case $b=\left(\begin{array}{l}s \\ 2\end{array}\right)-t$ for some $1 \leq t \leq \frac{s}{2}$}

Call a partition $U_{1}, \ldots, U_{k}$ of a finite set $X$ an equipartition if $\left\|U_{i}|-| U_{j}\right\| \leq 1$ for all $i \neq j$. Recall the Turán graph, $T_{s}(n)$, is the complete $s$-partite graph with $n$ vertices, whose parts form an equipartition of its vertex set.

Definition 6. Given integers $a \geq 2$ and $n \geq s \geq 1$, define $\mathbb{T}_{s, a}(n)$ to be the set of multigraphs $G=([n], w)$ with the following property. There is an equipartition $U_{1}, \ldots, U_{s}$ of $[n]$ such that

$$
w(x y)= \begin{cases}a-1 & \text { if } x y \in\left(\begin{array}{c}
U_{i} \\
2
\end{array}\right) \text { for some } i \in[s] . \\
a & \text { if }(x, y) \in U_{i} \times U_{j} \text { for some } i \neq j \in[s] .\end{cases}
$$

We think of elements of $\mathbb{T}_{s, a}(n)$ as multigraph analogues of Turán graphs. Let $t_{s}(n)$ be the number of edges in $T_{s}(n)$.

Theorem 4. Let $s, q, a, t$ be integers satisfying $a \geq 2, q=a\left(\begin{array}{l}s \\ 2\end{array}\right)-t$ and either

(a) $s \geq 2$ and $t=1$ or

(b) $s \geq 4$ and $2 \leq t \leq \frac{s}{2}$.

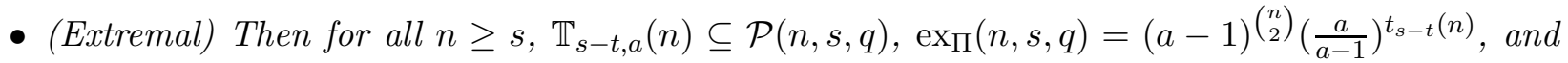
$\operatorname{ex}_{\Pi}(s, q)=(a-1)\left(\frac{a}{a-1}\right)^{\frac{s-t-1}{s-t}}$. If $(a)$ holds and $n \geq s$ or $(b)$ holds and $n$ is sufficiently large, then $\mathcal{P}(n, s, q)=\mathbb{T}_{s-t, a}(n)$.

- (Stability) For all $\delta>0$, there is $M$ and $\epsilon$ such that for all $n>M$ and $G \in F(n, s, q)$, if $P(G)>\operatorname{ex}_{\Pi}(n, s, q)^{1-\epsilon}$ then $G$ is $\delta$-close to an element of $\mathbb{T}_{s-t, a}(n)$.

\subsection{The case $(s, q)=(4,9)$}

The case $(s, q)=(4,9)$ is the first pair where $s \geq 2$ and $q \geq\left(\begin{array}{l}s \\ 2\end{array}\right)$ which is not covered by Theorems 3 and 4, and is closely related to an old question in extremal combinatorics. Let $\operatorname{ex}\left(n,\left\{C_{3}, C_{4}\right\}\right)$ denote the maximum number of edges in a graph on $n$ vertices which contains no $C_{3}$ or $C_{4}$ as a non-induced subgraph.

Theorem 5. $\operatorname{ex}_{\Pi}(n, 4,9)=2^{\operatorname{ex}\left(n,\left\{C_{3}, C_{4}\right\}\right)}$ for all $n \geq 4$.

It is known that

$$
\left(\frac{1}{2 \sqrt{2}}+o(1)\right) n^{3 / 2}<\operatorname{ex}\left(n,\left\{C_{3}, C_{4}\right\}\right)<\left(\frac{1}{2}+o(1)\right) n^{3 / 2}
$$

and an old conjecture of Erdös and Simonovits [4] states that the lower bound is correct.

The next case not covered here is $(s, q)=(4,15)$ and it was shown in [14] that $\operatorname{ex}_{\Pi}(n, 4,15)=$ $2^{\gamma n^{2}+o\left(n^{2}\right)}$ where $\gamma$ is transcendental and $2^{\gamma}$ is also transcendental if we assume Schanuel's conjecture from number theory. Many other cases were conjectured in [14 to have transcendental behaviour like the case $(4,15)$. This suggests that determining $\operatorname{ex}_{\Pi}(s, q)$ for all pairs $(s, q)$ will be a hard problem. 


\subsection{Enumeration and structure of most $(n, s, q)$-graphs}

Combining the extremal results of Theorems 3, 4, and 5 with Theorem 1 we obtain Theorem 6 below, which enumerates $F(n, s, q)$ for many cases of $(s, q)$.

Theorem 6. Let $s, q, a, b$ be integers satisfying $s \geq 2, a \geq 0$, and $q=a\left(\begin{array}{c}s \\ 2\end{array}\right)+b$.

(i) If $0 \leq b \leq s-2$, then $|F(n, s, q)|=(a+1)\left(\begin{array}{c}n \\ 2\end{array}\right) 2^{o\left(n^{2}\right)}$.

(ii) If $b=\left(\begin{array}{c}s \\ 2\end{array}\right)-t$ where $2 \leq t \leq \frac{s}{2}$, then $|F(n, s, q)|=(a+1)^{\left(\begin{array}{c}n \\ 2\end{array}\right)\left(\frac{a+2}{a+1}\right)^{t_{s-t}}(n)+o\left(n^{2}\right)}$.

(iii) $|F(n, 4,3)|=2^{\Theta\left(n^{3 / 2}\right)}$.

In our last main result, Theorem 7 below, we combine the stability results of Theorems 3 and 4 with Theorem 2 to prove approximate structure theorems for many $(s, q)$. Given $\delta>0$ and a set $E(n) \subseteq F(n, s, q)$, let $E^{\delta}(n)$ be the set of $G \in F(n, s, q)$ such that $G$ is $\delta$-close to some $G^{\prime} \in E(n)$.

Definition 7. Suppose $n, a, s$ are integers such that $n, s \geq 1$.

(i) If $a \geq 1$, set $U_{a}(n)=\left\{G=([n], w): G\right.$ is a submultigraph of some $\left.G^{\prime} \in \mathbb{U}_{a}(n)\right\}$.

(ii) If $a \geq 2$, set $T_{s, a}(n)=\left\{G=([n], w): G\right.$ is a submultigraph of some $\left.G^{\prime} \in \mathbb{T}_{s, a}(n)\right\}$.

Observe that in each case, $\mathbb{U}_{a}(n) \subseteq U_{a}(n)$ and $\mathbb{T}_{s, a}(n) \subseteq T_{s, a}(n)$.

Theorem 7. Suppose $s, q, a, t, b$ are integers such that $n \geq s \geq 2$, and $E(n)$ is a set of multigraphs such that one of the following holds.

(i) $a \geq 0, q=a\left(\begin{array}{l}s \\ 2\end{array}\right)+b$ for some $0 \leq b \leq s-2$, and $E(n)=U_{a}(n)$.

(ii) $a \geq 1, q=a\left(\begin{array}{l}s \\ 2\end{array}\right)-t$ for some $1 \leq t \leq \frac{s}{2}$, and $E(n)=T_{s-t, a}(n)$.

Then for all $\delta>0$ there exists $\beta>0$ such that for all sufficiently large $n$,

$$
\frac{\left|F(n, s, q) \backslash E^{\delta}(n)\right|}{|F(n, s, q)|} \leq 2^{-\beta\left(\begin{array}{c}
n \\
2
\end{array}\right)} .
$$

\section{Proof of Theorems 6 and 7}

In this section we prove Theorems 6 and 7 assuming Theorems 2, 3, and 4 .

Proof of Theorem 6. Suppose first that case (i) holds. By Theorem 3 (Extremal),

$$
\operatorname{ex}_{\Pi}\left(s, q+\left(\begin{array}{l}
s \\
2
\end{array}\right)\right)=\operatorname{ex}_{\Pi}\left(s,(a+1)\left(\begin{array}{l}
s \\
2
\end{array}\right)+b\right)=a+1 .
$$

If $a=0$, then $\operatorname{ex}_{\Pi}\left(s, q+\left(\begin{array}{l}s \\ 2\end{array}\right)\right)=1$, so Theorem 11 implies $|F(n, s, q)|=2^{o\left(n^{2}\right)}=(a+1)^{\left(\begin{array}{l}n \\ 2\end{array}\right)} 2^{o\left(n^{2}\right)}$. If $a \geq 1$, then $\operatorname{ex}_{\Pi}\left(s, q+\left(\begin{array}{l}s \\ 2\end{array}\right)\right)=a+1>1$, so Theorem 1 implies

$$
|F(n, s, q)|=(a+1)^{\left(\begin{array}{c}
n \\
2
\end{array}\right)+o\left(n^{2}\right)}=(a+1)^{\left(\begin{array}{c}
n \\
2
\end{array}\right)} 2^{o\left(n^{2}\right)} .
$$

Suppose now that case (ii) holds. So $q=a\left(\begin{array}{l}s \\ 2\end{array}\right)+\left(\begin{array}{l}s \\ 2\end{array}\right)-t=(a+1)\left(\begin{array}{l}s \\ 2\end{array}\right)-t$. By Theorem 4 (Extremal),

$$
\operatorname{ex}_{\Pi}\left(s, q+\left(\begin{array}{l}
s \\
2
\end{array}\right)\right)=\operatorname{ex}_{\Pi}\left(s,(a+2)\left(\begin{array}{l}
s \\
2
\end{array}\right)-t\right)=(a+1)\left(\frac{a+2}{a+1}\right)^{\frac{s-t-1}{s-t}} .
$$


Since $a \geq 0$, this shows $\operatorname{ex}_{\Pi}\left(s, q+\left(\begin{array}{l}s \\ 2\end{array}\right)\right)>1$, so Theorem 1 implies

$$
|F(n, s, q)|=\left((a+1)\left(\frac{a+2}{a+1}\right)^{\frac{s-t-1}{s-t}}\right)^{\left(\begin{array}{l}
n \\
2
\end{array}\right)+o\left(n^{2}\right)}=(a+1)^{\left(\begin{array}{c}
n \\
2
\end{array}\right)}\left(\frac{a+2}{a+1}\right)^{t_{s-t}(n)+o\left(n^{2}\right)} .
$$

For (iii) first observe that any subgraph of a graph of girth at least 5 is a $(4,3)$-graph, and since $\operatorname{ex}\left(n,\left\{C_{3}, C_{4}\right\}\right) \geq c_{1} n^{3 / 2}$ for some constant $c_{1}>0$ (see [4]) we obtain the lower bound. For the upper bound, observe that in a $(4,3)$-graph, there is at most one pair with multiplicity at least two and the set of pairs with multiplicity one forms a graph with no $C_{4}$. By the Kleitman-Winston theorem [12], the number of ways to choose the pairs of multiplicity one is at most $2^{c_{2} n^{3 / 2}}$ for some constant $c_{2}>0$ and this gives the upper bound.

Proof of Theorem 7. Fix $\delta>0$. Observe that if case (i) holds (respectively, case (ii)), then $\left(s, q+\left(\begin{array}{l}s \\ 2\end{array}\right)\right)$ satisfies the hypotheses of Theorem 3 (respectively, Theorem 4). Let

$$
\mathbb{E}(n)= \begin{cases}\mathbb{U}_{a+1}(n) & \text { in case (i) } \\ \mathbb{T}_{s-t, a+1}(n) & \text { in case (ii) }\end{cases}
$$

By Theorem 3 (Stability) in case (i) and Theorem 4 (Stability) in case(ii), there is $\epsilon>0$ so that for sufficiently large $n$, if $G^{+} \in F\left(n, s, q+\left(\begin{array}{l}s \\ 2\end{array}\right)\right)$ satisfies $P\left(G^{+}\right)>\operatorname{ex}_{\Pi}\left(n, s, q+\left(\begin{array}{l}s \\ 2\end{array}\right)\right)^{1-\epsilon}$, then $G^{+}$is $\delta$-close to some $G^{\prime} \in \mathbb{E}(n)$. Note that $G^{\prime} \in \mathbb{E}(n)$ implies there is $H \in E(n)$ such that $H^{+}=G$. Combining this our choice of $\epsilon$, we obtain the following. For all sufficienlty large $n$ and $G \in F(n, s, q)$,

$$
\text { if } P\left(G^{+}\right)>\operatorname{ex}_{\Pi}\left(n, s, q+\left(\begin{array}{l}
s \\
2
\end{array}\right)\right)^{1-\epsilon} \text {, then } G^{+} \text {is } \delta \text {-close to } H^{+} \text {, for some } H \in E(n) \text {. }
$$

By Theorem 3 (Extremal) in case (i) and Theorem 4 (Extremal) in case(ii), we must have that $\operatorname{ex}_{\Pi}\left(s, q+\left(\begin{array}{l}s \\ 2\end{array}\right)\right)>1$. So Theorem 2 implies there is $\beta>0$ such that for all sufficiently large $n$ the following holds.

$$
\frac{|F(n, s, q) \backslash E(n, s, q, \epsilon)|}{|F(n, s, q)|} \leq 2^{-\beta n^{2}}
$$

So to show (2), it suffices to show that for sufficiently large $n, E(n, s, q, \epsilon) \subseteq E^{\delta}(n)$. Fix $n$ sufficiently large and suppose $G=\left([n], w^{G}\right) \in E(n, s, q, \epsilon)$. By definition, this means there is $G^{\prime} \in F(n, s, q)$ such that $P\left(G^{\prime+}\right)>\operatorname{ex}_{\Pi}\left(n, s, q+\left(\begin{array}{c}s \\ 2\end{array}\right)\right)^{1-\epsilon}$ and $G$ is a submultigraph of $G^{\prime}$. By (3), $G^{\prime+}$ is $\delta$ close to $H^{+}$, for some $H \in E(n)$. Define $H^{\prime}=\left([n], w^{H^{\prime}}\right)$ such that $w^{H^{\prime}}(x y)=w^{G}(x y)$ if $x y \in$ $\left(\begin{array}{c}{[n]} \\ 2\end{array}\right) \backslash \Delta\left(G^{\prime}, H\right)$, and $w^{H^{\prime}}(x y)=0$ if $x y \in \Delta\left(G^{\prime}, H\right)$. We claim $H^{\prime}$ is a submultigraph of $H$. Fix $x y \in\left(\begin{array}{c}{[n]} \\ 2\end{array}\right)$. We want to show $w^{H^{\prime}}(x y) \leq w^{H}(x y)$. If $x y \in \Delta\left(G^{\prime}, H\right)$, then $w^{H^{\prime}}(x y)=0 \leq w^{H}(x y)$ is immediate. If $x y \notin \Delta\left(G^{\prime}, H\right)$, then $w^{H^{\prime}}(x y)=w^{G}(x y) \leq w^{G^{\prime}}(x y)=w^{H}(x y)$, where the inequality is because $G$ is a submultigraph of $G^{\prime}$ and the last equality is because $x y \notin \Delta\left(G^{\prime}, H\right)$. Thus $H^{\prime}$ is a submultigraph of $H \in E(n)$, which implies $H^{\prime}$ is also in $E(n)$. By definition of $H^{\prime}$, $\Delta\left(G, H^{\prime}\right) \subseteq \Delta\left(G^{\prime}, H\right)=\Delta\left(G^{\prime+}, H^{+}\right)$. Since $G^{\prime+}$ and $H^{+}$are $\delta$-close, this implies $\left|\Delta\left(G, H^{\prime}\right)\right| \leq \delta n^{2}$, and $G \in E^{\delta}(n)$.

\section{Proof of Theorem 2}

In this section we prove Theorem 2, We will use Theorem 8 below, which is a version of the hypergraph containers theorem of [1,15] for multigraphs. Theorem 8 was proved in [14]. 
Definition 8. Suppose $s \geq 2$ and $q \geq 0$ are integers. Set

$$
\mathcal{H}(s, q)=\{G=([s], w): \mu(G) \leq q \text { and } S(G)>q\}, \quad \text { and } \quad g(s, q)=|\mathcal{H}(s, q)| .
$$

If $G=(V, w)$ is a multigraph, let $\mathcal{H}(G, s, q)=\left\{X \in\left(\begin{array}{l}V \\ s\end{array}\right): G[X] \cong G^{\prime}\right.$ for some $\left.G^{\prime} \in \mathcal{H}(s, q)\right\}$.

Theorem 8. For every $0<\delta<1$ and integers $s \geq 2, q \geq 0$, there is a constant $c=c(s, q, \delta)>0$ such that the following holds. For all sufficiently large $n$, there is $\mathcal{G}$ a collection of multigraphs of multiplicity at most $q$ and with vertex set $[n]$ such that

(i) for every $J \in F(n, s, q)$, there is $G \in \mathcal{G}$ such that $J$ is a submultigraph of $G$,

(ii) for every $G \in \mathcal{G},|\mathcal{H}(G, s, q)| \leq \delta\left(\begin{array}{l}n \\ s\end{array}\right)$, and

(iii) $\log |\mathcal{G}| \leq c n^{2-\frac{1}{4 s}} \log n$.

We will also use the following two results appearing in [14].

Lemma 1 (Lemma 1 of [14]). Fix integers $s \geq 2$ and $q \geq 0$. For all $0<\nu<1$, there is $0<\delta<1$ such that for all sufficiently large $n$, the following holds. If $G=([n], w)$ satisfies $\mu(G) \leq q$ and $|\mathcal{H}(G, s, q)| \leq \delta\left(\begin{array}{l}n \\ 2\end{array}\right)$, then $G$ is $\nu$-close to some $G^{\prime}$ in $F(n, s, q)$.

Proposition 1 (Proposition 2 in [14]). For all $n \geq s \geq 2$ and $q \geq 0$, $\operatorname{ex}_{\Pi}(s, q)$ exists and

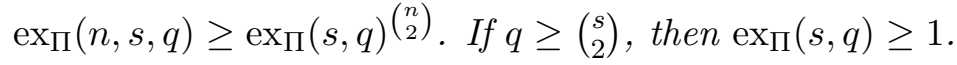

Proof of Theorem 2, Fix $\epsilon>0$ and set $\nu=\left(\epsilon \log \left(\operatorname{ex}_{\Pi}\left(s, q+\left(\begin{array}{l}s \\ 2\end{array}\right)\right)\right) /(8 \log (q+1))\right.$. Choose $\delta>0$ according to Lemma 1 so that the following holds for all sufficiently large $n$.

$$
\text { Any } G=([n], w) \text { with } \mu(G) \leq q \text { and }|\mathcal{H}(G, s, q)| \leq \delta\left(\begin{array}{l}
n \\
2
\end{array}\right) \text { is } \nu \text {-close to some } G^{\prime} \text { in } F(n, s, q) \text {. }
$$

Fix $n$ sufficiently large. Apply Theorem 8 to obtain a constant $c$ and a collection $\mathcal{G}$ of multigraphs of multiplicity at most $q$ and with vertex set $[n]$ satisfying (i)-(iii) of Theorem 8 . Suppose that $H=\left([n], w^{H}\right) \in F(n, s, q) \backslash E(n, s, q, \epsilon)$. By (i), there is $G=\left([n], w^{G}\right) \in \mathcal{G}$ such that $H$ is a submultigraph of $G$ and $|\mathcal{H}(G, s, q)| \leq \delta\left(\begin{array}{l}n \\ s\end{array}\right)$. We claim that $P\left(G^{+}\right) \leq \operatorname{ex}_{\Pi}\left(n, s, q+\left(\begin{array}{l}s \\ 2\end{array}\right)\right)^{1-\epsilon / 2}$. Suppose towards a contradiction this is not the case, so $P\left(G^{+}\right)>\operatorname{ex}_{\Pi}\left(n, s, q+\left(\begin{array}{l}s \\ 2\end{array}\right)\right)^{1-\epsilon / 2}$. By (44), $|\mathcal{H}(G, s, q)| \leq \delta\left(\begin{array}{l}n \\ s\end{array}\right)$ implies there is $G^{\prime}=\left([n], w^{G^{\prime}}\right) \in F(n, s, q)$ which is $\nu$-close to $G$. Define $H^{\prime}=\left([n], w^{H^{\prime}}\right)$ by setting $w^{H^{\prime}}(x y)=w^{H}(x y)$ for all $x y \in\left(\begin{array}{c}{[n]} \\ 2\end{array}\right) \backslash \Delta\left(G, G^{\prime}\right)$ and $w^{H^{\prime}}(x y)=0$ for all $x y \in \Delta\left(G, G^{\prime}\right)$. By construction and because $H^{\prime}$ is a submultigraph of $G^{\prime}$, we have that $H$ is also a submultigraph of $G^{\prime}$. Observe

$$
P\left(G^{\prime+}\right)=P\left(G^{+}\right)\left(\prod_{x y \in \Delta\left(H, H^{\prime}\right)} \frac{w^{G^{\prime}}(x y)+1}{w^{G}(x y)+1}\right) \geq P\left(G^{+}\right)(q+1)^{-\left|\Delta\left(G, G^{\prime}\right)\right|},
$$

where the inequality is because $1 \leq w^{G^{\prime}}(x y)+1, w^{G}(x y)+1 \leq q+1$ implies $\frac{w^{G^{\prime}}(x y)+1}{w^{G}(x y)+1} \geq \frac{1}{q+1}$. Combining this with the fact that $G$ and $G^{\prime}$ are $\nu$-close, the definition of $\nu$, and our assumption that $P\left(G^{+}\right) \geq \operatorname{ex}_{\Pi}\left(n, s, q+\left(\begin{array}{c}s \\ 2\end{array}\right)\right)^{1-\epsilon / 2}$, we have that $P\left(G^{\prime+}\right)$ is at least the following.

$P\left(G^{+}\right)(q+1)^{-\nu n^{2}}=P\left(G^{+}\right) \operatorname{ex} \Pi\left(s, q+\left(\begin{array}{l}s \\ 2\end{array}\right)\right)^{-\epsilon n^{2} / 8} \geq \operatorname{ex}_{\Pi}\left(n, s, q+\left(\begin{array}{l}s \\ 2\end{array}\right)\right)^{1-\epsilon / 2} \operatorname{ex}_{\Pi}\left(s, q+\left(\begin{array}{l}s \\ 2\end{array}\right)\right)^{-\epsilon n^{2} / 8}$. 
Since $\operatorname{ex}_{\Pi}\left(n, s, q+\left(\begin{array}{l}s \\ 2\end{array}\right)\right)^{1 /\left(\begin{array}{l}n \\ 2\end{array}\right)} \geq \operatorname{ex}_{\Pi}\left(s, q+\left(\begin{array}{l}s \\ 2\end{array}\right)\right.$ ) (see Proposition 1), we obtain that the right hand side is at least

$$
\operatorname{ex}_{\Pi}\left(n, s, q+\left(\begin{array}{l}
s \\
2
\end{array}\right)\right)^{1-\epsilon / 2} \operatorname{ex}\left(n, s, q+\left(\begin{array}{l}
s \\
2
\end{array}\right)\right)^{-\epsilon n^{2} /\left(8\left(\begin{array}{l}
n \\
2
\end{array}\right)\right)} \geq \operatorname{ex}_{\Pi}\left(n, s, q+\left(\begin{array}{l}
s \\
2
\end{array}\right)\right)^{1-\epsilon}
$$

where the inequality is because $n$ large implies $\epsilon n^{2} /\left(8\left(\begin{array}{c}n \\ 2\end{array}\right)\right) \leq \epsilon / 2$. But now $H$ is a submultigraph of $G^{\prime}$ and $P\left(G^{\prime+}\right) \geq \operatorname{ex}_{\Pi}\left(n, s, q+\left(\begin{array}{l}s \\ 2\end{array}\right)\right)^{1-\epsilon}$, contradicting that $H \in F(n, s, q) \backslash E(n, s, q, \epsilon)$. Therefore, every element of $F(n, s, q) \backslash E(n, s, q, \epsilon)$ can be constructed as follows.

- Choose some $G \in \mathcal{G}$ with $P\left(G^{+}\right) \leq \operatorname{ex}_{\Pi}\left(n, s, q+\left(\begin{array}{l}s \\ 2\end{array}\right)\right)^{1-\epsilon / 2}$. There are at most $c n^{2-\frac{1}{4 s}} \log n$ choices. Since $n$ is large and $\operatorname{ex}_{\Pi}\left(s, q+\left(\begin{array}{c}s \\ 2\end{array}\right)\right)>1$, we may assume $c n^{2-\frac{1}{4 s}} \log n \leq \operatorname{ex}_{\Pi}\left(s, q+\left(\begin{array}{l}s \\ 2\end{array}\right)\right)^{\epsilon}\left(\begin{array}{l}n \\ 2\end{array}\right) / 4$.

- Choose a submultigraph of $G$. There are at most $P\left(G^{+}\right) \leq \operatorname{ex}_{\Pi}\left(n, s, q+\left(\begin{array}{l}s \\ 2\end{array}\right)\right)^{1-\epsilon / 2}$ choices.

This shows

$$
\begin{aligned}
|F(n, s, q) \backslash E(n, s, q, \epsilon)| & \leq \operatorname{ex}_{\Pi}\left(s, q+\left(\begin{array}{l}
s \\
2
\end{array}\right)\right)^{\epsilon\left(\begin{array}{c}
n \\
2
\end{array}\right) / 4} \operatorname{ex}_{\Pi}\left(n, s, q+\left(\begin{array}{l}
s \\
2
\end{array}\right)\right)^{1-\epsilon / 2} \\
& \leq \operatorname{ex}_{\Pi}\left(s, q+\left(\begin{array}{l}
s \\
2
\end{array}\right)\right)^{-\epsilon\left(\begin{array}{c}
n \\
2
\end{array}\right) / 4} \operatorname{ex}_{\Pi}\left(n, s, q+\left(\begin{array}{l}
s \\
2
\end{array}\right)\right)
\end{aligned}
$$

where the second inequality is because $\operatorname{ex}_{\Pi}\left(n, s, q+\left(\begin{array}{l}s \\ 2\end{array}\right)\right) \geq \operatorname{ex}_{\Pi}\left(s, q+\left(\begin{array}{l}s \\ 2\end{array}\right)\right)\left(\begin{array}{l}n \\ 2\end{array}\right)$. By Theorem 1 , $|F(n, s, q)| \geq \operatorname{ex}_{\Pi}(n, s, q)$, so this implies

$$
\frac{|F(n, s, q) \backslash E(n, s, q, \epsilon)|}{|F(n, s, q)|} \leq \operatorname{ex}_{\Pi}\left(s, q+\left(\begin{array}{l}
s \\
2
\end{array}\right)\right)^{-\epsilon\left(\begin{array}{c}
n \\
2
\end{array}\right) / 4} .
$$

Setting $\beta=\frac{\epsilon}{4} \log _{2}\left(\operatorname{ex}_{\Pi}\left(s, q+\left(\begin{array}{c}s \\ 2\end{array}\right)\right)\right.$ finishes the proof $\left(\right.$ note $\beta>0$ since $\left.\operatorname{ex}_{\Pi}\left(s, q+\left(\begin{array}{l}s \\ 2\end{array}\right)\right)>1\right)$.

\section{$5 \quad$ Extremal Results}

In this section we prove the extremal statements in Theorems 3 and 4 . We begin with some preliminaries. Suppose $s \geq 2$ and $q \geq\left(\begin{array}{l}s \\ 2\end{array}\right)$. It was shown in [9] that $\operatorname{ex}_{\Sigma}(s, q)$ exists, and the AM-GM inequality implies that

$$
\operatorname{ex}_{\Pi}(s, q)=\lim _{n \rightarrow \infty} \operatorname{ex}_{\Pi}(n, s, q)^{1 /\left(\begin{array}{c}
n \\
2
\end{array}\right)} \leq \lim _{n \rightarrow \infty} \frac{\operatorname{ex}_{\Sigma}(n, s, q)}{\left(\begin{array}{l}
n \\
2
\end{array}\right)}=\operatorname{ex}_{\Sigma}(s, q) .
$$

The following lemma is an integer version of the AM-GM inequality.

Lemma 2. If $\ell \geq 2, k \in[\ell]$ and $a, x_{1}, \ldots, x_{\ell}$ are positive integers such that $\sum_{i=1}^{\ell} x_{i} \leq a \ell-k$, then $\prod_{i=1}^{\ell} x_{i} \leq a^{\ell-k}(a-1)^{k}$. Moreover, equality holds if and only if exactly $k$ of the $x_{i}$ are equal to $a-1$ and the rest are equal to $a$.

Proof. If there are $x_{i}$ and $x_{j}$ with $x_{i}<x_{j}-1$, then replacing $x_{i}$ with $x_{i}+1$ and replacing $x_{j}$ with $x_{j}-1$ increases the product and keeps the sum unchanged. So no two of the $x_{i}$ 's differ by more than one when the product is maximized.

Corollary 1. Let $n \geq s \geq 2, a \geq 2$, and $(a-1)\left(\begin{array}{c}s \\ 2\end{array}\right) \leq q<a\left(\begin{array}{c}s \\ 2\end{array}\right)$. Suppose $G \in \mathcal{S}(n, s, q)$ has all edge multiplicities in $\{a, a-1\}$ and contains exactly $k$ edges of multiplicity $a-1$. Then for all other $G^{\prime} \in F(n, s, q), G^{\prime} \in \mathcal{P}(n, s, q)$ if and only if $G^{\prime}$ has $k$ edges of multiplicity $a-1$ and all other edges of multiplicity a. Consequently, $G \in \mathcal{P}(n, s, q) \subseteq \mathcal{S}(n, s, q)$. 
Proof. Fix $G$ so that the hypotheses hold. Then $S(G)=a\left(\begin{array}{c}n \\ 2\end{array}\right)-k$ and $P(G)=a^{\left(\begin{array}{c}n \\ 2\end{array}\right)-k}(a-1)^{k}$. Let $G^{\prime}=([n], w)$ be another element of $F(n, s, q)$. Since $G \in \mathcal{S}(n, s, q)$, we have

$$
S\left(G^{\prime}\right) \leq S(G)=a\left(\begin{array}{l}
n \\
2
\end{array}\right)-k
$$

By Lemma 2 with $\ell=\left(\begin{array}{c}n \\ 2\end{array}\right), P\left(G^{\prime}\right) \leq a^{\left(\begin{array}{c}n \\ 2\end{array}\right)-k}(a-1)^{k}$ with equality if and only if $\left\{w(x y): x y \in\left(\begin{array}{c}{[n]} \\ 2\end{array}\right)\right\}$ consists of $k$ elements equal to $a-1$ and the rest equal to $a$. This shows $G^{\prime} \in \mathcal{P}(n, s, q)$ if and only if $G^{\prime}$ has $k$ edges of multiplicity $a-1$ and the rest of multiplicity $a$. Consequently, $G \in \mathcal{P}(n, s, q)$. To show $\mathcal{P}(n, s, q) \subseteq \mathcal{S}(n, s, q)$, let $G^{\prime} \in \mathcal{P}(n, s, q)$. Then by what we have shown, $S\left(G^{\prime}\right)=a\left(\begin{array}{l}n \\ 2\end{array}\right)-k=S(G)$, so $G \in \mathcal{S}(n, s, q)$ implies $G^{\prime} \in \mathcal{S}(n, s, q)$.

The following is a consequence of Theorem 5.2 in [2] (case $b=0$ ) and Theorems 8 and 9 in [9] (cases $0<b \leq s-2$ ).

Theorem 9 (Bondy-Tuza [2], Füredi-Kündgen [9]). Let $n \geq s \geq 2, a \geq 1,0 \leq b \leq s-2$, and $q=a\left(\begin{array}{l}s \\ 2\end{array}\right)+b$. Then

$$
\operatorname{ex}_{\Sigma}(n, s, q) \leq a\left(\begin{array}{l}
n \\
2
\end{array}\right)+\left\lfloor\frac{b}{b+1} n\right\rfloor .
$$

with equality holding when $b=s-2$ and when $b=0$.

Proof of Theorem 3 (Extremal). Since $\mathbb{U}_{a}(n) \in F(n, s, q), a^{\left(\begin{array}{c}n \\ 2\end{array}\right)} \leq \operatorname{ex}_{\Pi}(n, s, q)$. On the other hand, let $G \in F(n, s, q)$. Theorem 9 implies that $S(G) \leq a\left(\begin{array}{c}n \\ 2\end{array}\right)+\left\lfloor\frac{b}{b+1} n\right\rfloor$. This along with Lemma 2 implies that $P(G) \leq a^{\left(\begin{array}{c}n \\ 2\end{array}\right)}((a+1) / a)^{\left\lfloor\frac{b}{b+1} n\right\rfloor}$. Thus $a^{\left(\begin{array}{c}n \\ 2\end{array}\right)} \leq \operatorname{ex}_{\Pi}(n, s, q) \leq a^{\left(\begin{array}{c}n \\ 2\end{array}\right)}((a+1) / a)^{\left\lfloor\frac{b}{b+1} n\right\rfloor}$, which implies $\operatorname{ex}_{\Pi}(s, q)=a$.

Case (a): If $b=0$, then Theorem 9 implies $\mathbb{U}_{a}(n) \in \mathcal{S}(n, s, q)$. Because $\mathbb{U}_{a}(n)$ has all edge multiplicities in $\{a\}$, Corollary 1 implies $\mathbb{U}_{a}(n) \in \mathcal{P}(n, s, q)$ and moreover, every other element of

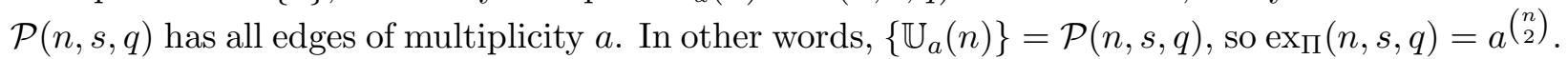

Case (b): If $b=s-2$, then it is straightforward to check $\mathbb{U}_{s-1, a}(n) \subseteq F(n, s, q)$. Since $S(G)=a\left(\begin{array}{l}n \\ 2\end{array}\right)+\left\lfloor\frac{s-2}{s-1} n\right\rfloor$ for all $G \in U_{s-1, a}(n)$, Theorem 9 implies $\mathbb{U}_{s-1, a}(n) \subseteq \mathcal{S}(n, s, q)$. Because every element in $\mathbb{U}_{s-1, a}(n)$ has all edge multiplicities in $\{a+1, a\}$, Corollary 1 implies $\mathbb{U}_{s-1, a}(n) \subseteq$ $\mathcal{P}(n, s, q)$ and every $G^{\prime} \in \mathcal{P}(n, s, q)$ contains exactly $\left\lfloor\frac{s-2}{s-1} n\right\rfloor$ edges of multiplicity $a+1$, and all others

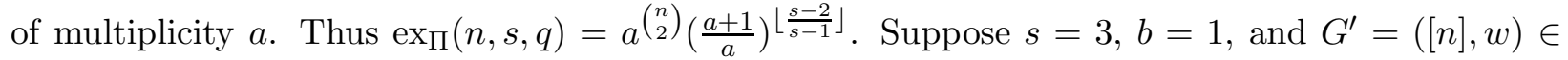
$\mathcal{P}(n, s, q)$. If there are $x, y \neq z \in[n]$ such that $w(x y)=w(x z)=a+1$, then because $G^{\prime}$ contains only edges of multiplicity $a+1$ and $a, S(\{x, y, z\}) \geq 2(a+1)+a=3 a+2>q$, a contradiction. Thus the edges of multiplicity $a+1$ form a matching of size $\left\lfloor\frac{n}{2}\right\rfloor$ in $G^{\prime}$, so $G^{\prime} \in \mathbb{U}_{s-1, a}(n)$. This shows $\mathbb{U}_{s-1, a}(n)=\mathcal{P}(n, s, q)$.

The following is a consequence of Theorem 5.2 of [2].

Theorem 10 (Bondy-Tuza [2]). Suppose $n \geq s \geq 2, a \geq 1$, and $q=a\left(\begin{array}{l}s \\ 2\end{array}\right)-1$. Then

$$
\operatorname{ex}_{\Sigma}(n, s, q)=(a-1)\left(\begin{array}{l}
n \\
2
\end{array}\right)+t_{s-1}(n) .
$$

Proof of Theorem 4(a) (Extremal). Since $\mathbb{T}_{s-1, a}(n) \subseteq F(n, s, q)$ and for all $G \in \mathbb{T}_{s-1, a}(n)$, $S(G)=(a-1)\left(\begin{array}{l}n \\ 2\end{array}\right)+t_{s-1}(n)$, Theorem 10 implies that $\mathbb{T}_{s-1, a}(n) \subseteq \mathcal{S}(n, s, q)$. Therefore Corollary 1 implies $\mathbb{T}_{s-1, a}(n) \subseteq \mathcal{P}(n, s, q)$ and each $G \in \mathcal{P}(n, s, q)$ has $t_{s-1}(n)$ edges of multiplicity $a$ and the 
rest of multiplicity $a-1$. Fix $G=([n], w) \in \mathcal{P}(n, s, q)$ and let $G^{\prime}$ be the graph with vertex set $[n]$ and edge set $E=\left\{x y \in\left(\begin{array}{c}{[n]} \\ 2\end{array}\right): w(x y)=a\right\}$. Then $G^{\prime}$ is $K_{s}$-free and has $t_{s-1}(n)$ edges, so by Turán's theorem, $G^{\prime}=T_{s-1}(n)$ and thus $G \in \mathbb{T}_{s-1, a}(n)$. So we have shown, $\mathcal{P}(n, s, q)=\mathbb{T}_{s-1, a}(n)$.

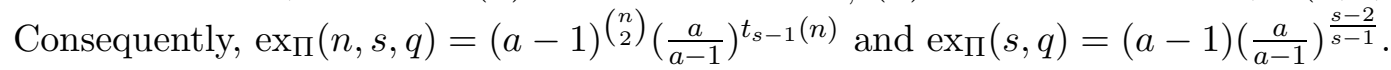

To prove Theorem 4(b) (Extremal), we will need the following theorem, as well as a few lemmas.

Theorem 11. [Dirac [3], Bondy-Tuza [2]] Let $n \geq s \geq 4, a \geq 1$, and $q=a\left(\begin{array}{c}s \\ 2\end{array}\right)-t$ for some $2 \leq t \leq \frac{s}{2}$. Then $\operatorname{ex}_{\Sigma}(n, s, q)=\operatorname{ex}_{\Sigma}\left(n, s^{\prime}, q^{\prime}\right)$ where $s^{\prime}=s-t+1$ and $q^{\prime}=a\left(\begin{array}{c}s^{\prime} \\ 2\end{array}\right)-1$.

Proof. Let $n \geq s \geq 4$ and $2 \leq t \leq s / 2$. In [3], Dirac proved that $\operatorname{ex}_{\Sigma}\left(n, s,\left(\begin{array}{c}s \\ 2\end{array}\right)-t\right)=t_{s-t}(n)$. This along with Lemma 5.1 in [2] implies that for all $a \geq 1$,

$\operatorname{ex}_{\Sigma}\left(n, s, a\left(\begin{array}{l}s \\ 2\end{array}\right)-t\right)=\operatorname{ex}_{\Sigma}\left(n, s,\left(\begin{array}{l}s \\ 2\end{array}\right)-t\right)+(a-1)\left(\begin{array}{l}n \\ 2\end{array}\right)=t_{s-t}(n)+(a-1)\left(\begin{array}{l}n \\ 2\end{array}\right)=\operatorname{ex}_{\Sigma}\left(n, s^{\prime}, a\left(\begin{array}{l}s^{\prime} \\ 2\end{array}\right)-1\right)$,

where the last equality is by Theorem 10 applied to $s^{\prime}$ and $a\left(\begin{array}{c}s^{\prime} \\ 2\end{array}\right)-1$.

Lemma 3. If $s, q, a, t$ are integers satisfying case (b) of Theorem 4, and $s^{\prime}=s-t+1, q^{\prime}=a\left(\begin{array}{c}s^{\prime} \\ 2\end{array}\right)-1$, then for all $n \geq s, \mathbb{T}_{s^{\prime}-1}(n) \subseteq \mathcal{P}(n, s, q)$ and $\operatorname{ex}_{\Pi}(n, s, q)=\operatorname{ex}_{\Pi}\left(n, s^{\prime}, q^{\prime}\right)$.

Proof. Set $s^{\prime}=s-t+1$ and $q^{\prime}=a\left(\begin{array}{c}s^{\prime} \\ 2\end{array}\right)-1$, and fix $n \geq s$. Fix $G \in \mathbb{T}_{s^{\prime}-1, a}(n)$. It is straightforward to check that $G \in F(n, s, q)$. By Theorem 11, $\operatorname{ex}_{\Sigma}\left(n, s^{\prime}, q^{\prime}\right)=\operatorname{ex}_{\Sigma}(n, s, q)$. Since $S(G)=(a-1)\left(\begin{array}{l}n \\ 2\end{array}\right)+$ $t_{s^{\prime}-1}(n)$, by Theorem [10 applied to $s^{\prime}$ and $q^{\prime}$, we have that $S(G)=\operatorname{ex}_{\Sigma}\left(n, s^{\prime}, q^{\prime}\right)=\operatorname{ex}_{\Sigma}(n, s, q)$. This shows $G \in \mathcal{S}(n, s, q)$. By Corollary 1, since $G$ has all edge multiplicities in $\{a, a-1\}, G \in$ $\mathcal{P}(n, s, q)$, so $P(G)=\operatorname{ex}_{\Pi}(n, s, q)$. Since $G \in \mathbb{T}_{s^{\prime}-1, a}(n)$ and $\mathbb{T}_{s^{\prime}-1, a}(n) \subseteq \mathcal{P}\left(n, s^{\prime}, q^{\prime}\right)$ by Theorem 4 (a) (Extremal), $P(G)=\operatorname{ex}_{\Pi}\left(n, s^{\prime}, q^{\prime}\right)$. Thus $\operatorname{ex}_{\Pi}(n, s, q)=P(G)=\operatorname{ex}_{\Pi}\left(n, s^{\prime}, q^{\prime}\right)$.

We now fix some notation. Given $n \in \mathbb{N}, z \in[n], Y \subseteq[n]$, and $G=([n], w)$, set

$$
S(Y)=\sum_{x y \in\left(\begin{array}{c}
Y \\
2
\end{array}\right)} w(x y), \quad S_{z}(Y)=\sum_{y \in Y} w(y z), \quad P(Y)=\prod_{x y \in\left(\begin{array}{c}
Y \\
2
\end{array}\right)} w(x y), \quad \text { and } \quad P_{z}(Y)=\prod_{y \in Y} w(y z)
$$

If $X \subseteq[n]$ is disjoint from $Y$, set $P(X, Y)=\prod_{x \in X, y \in Y} w(x y)$.

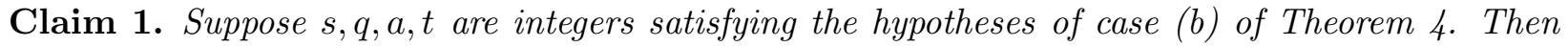
for all $n \geq 2 s$ and $s-t+1 \leq y \leq s-1$,

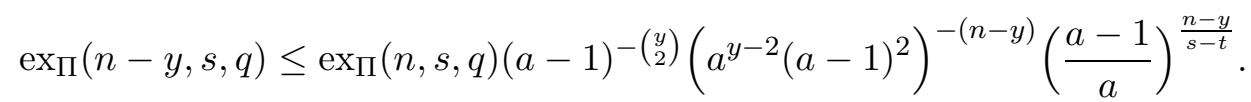

Proof. Set $s^{\prime}=s-t+1$ and $q^{\prime}=a\left(\begin{array}{c}s^{\prime} \\ 2\end{array}\right)-1$. Fix $n \geq s$ and $s^{\prime} \leq y \leq s-1$. Choose some $H=([n-y], w) \in \mathbb{T}_{s^{\prime}-1, a}(n-y)$ and let $U_{1}, \ldots, U_{s^{\prime}-1}$ be the partition of $[n-y]$ corresponding to $H$. Observe that there is some $i$ such that $\left|U_{i}\right| \geq \frac{n-y}{s^{\prime}-1}$. Without loss of generality, assume $\left|U_{1}\right| \geq \frac{n-y}{s^{\prime}-1}$. Assign the elements of $Y^{\prime}:=[n] \backslash[n-y]$ to the $U_{i}$ in as even a way as possible, to obtain an equipartition $U_{1}^{\prime}, \ldots, U_{s^{\prime}-1}^{\prime}$ of $[n]$ extending $U_{1}, \ldots, U_{s^{\prime}-1}$. Observe that because $s^{\prime} \leq\left|Y^{\prime}\right| \leq s-1$ and $s^{\prime}-1=s-t \geq s / 2$, for each $i,\left|U_{i}^{\prime} \backslash U_{i}\right| \in\{1,2\}$, and there is at least one $i$ such that $\left|U_{i}^{\prime} \backslash U_{i}\right|=1$. Since $\left|U_{1}\right| \geq \frac{n-y}{s-t}$, by redistributing $Y^{\prime}$ if necessary, we may assume that $\left|U_{1}^{\prime} \backslash U_{1}\right|=1$. Define a new multigraph $H^{\prime}=\left([n], w^{\prime}\right)$ so that $w^{\prime}(x y)=a-1$ if $x y \in\left(\begin{array}{c}U_{i}^{\prime} \\ 2\end{array}\right)$ for some $i \in\left[s^{\prime}-1\right]$ and $w^{\prime}(x y)=a$ if $(x, y) \in U_{i}^{\prime} \times U_{j}^{\prime}$ for some $i \neq j$. Note that by construction $H^{\prime} \in \mathbb{T}_{s^{\prime}-1, a}(n)$ and 
$H^{\prime}[[n-y]]=H$. By Lemma 3, since $n-y \geq s, H \in \mathbb{T}_{s^{\prime}-1, a}(n-y)$ and $H^{\prime} \in \mathbb{T}_{s^{\prime}-1, a}(n)$ imply $H \in \mathcal{P}(n-y, s, q)$ and $H^{\prime} \in \mathcal{P}(n, s, q)$. These facts imply the following.

$$
\operatorname{ex}_{\Pi}(n, s, q)=P\left(H^{\prime}\right)=P(H) P\left(Y^{\prime}\right) P\left(Y^{\prime},[n-y]\right)=\operatorname{ex}_{\Pi}(n-y, s, q) P\left(Y^{\prime}\right) P\left(Y^{\prime},[n-y]\right) .
$$

By definition of $H^{\prime}$, if $\left|U_{i}^{\prime} \backslash U_{i}\right|=2$, then for all $z \in U_{i}, P_{z}\left(Y^{\prime}\right)=a^{y-2}(a-1)^{2}$ and if $\left|U_{i}^{\prime} \backslash U_{i}\right|=1$, then for all $z \in U_{i}, P_{z}\left(Y^{\prime}\right)=a^{y-1}(a-1)$. Since $\left|U_{1}^{\prime} \backslash U_{1}\right|=1$, this implies

$$
P\left(Y^{\prime},[n-y]\right) \geq\left(a^{y-2}(a-1)^{2}\right)^{n-y-\left|U_{1}\right|}\left(a^{y-1}(a-1)\right)^{\left|U_{1}\right|}=\left(a^{y-2}(a-1)^{2}\right)^{n-y}\left(\frac{a}{a-1}\right)^{\left|U_{1}\right|} .
$$

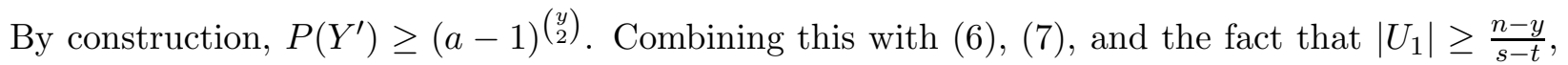
we obtain

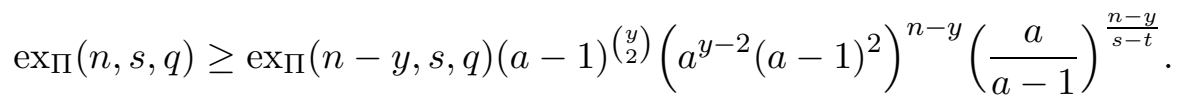

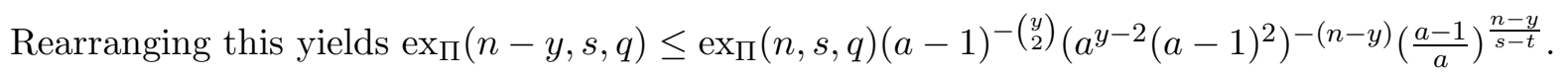

Lemma 4. Let $n \geq s \geq 4, a \geq 2$, and $q=a\left(\begin{array}{c}s \\ 2\end{array}\right)-t$ for some $2 \leq t \leq \frac{s}{2}$. Suppose $G \in F(n, s, q)$ and $Y \in\left(\begin{array}{c}{[n]} \\ s-t+1\end{array}\right)$ satisfies $S(Y) \geq a\left(\begin{array}{c}s-t+1 \\ 2\end{array}\right)$. Then there is $Y \subseteq Y^{\prime} \subseteq[n]$ such that $s-t+1 \leq\left|Y^{\prime}\right| \leq s-1$ and for all $z \in[n] \backslash Y^{\prime}, S_{z}\left(Y^{\prime}\right) \leq a\left|Y^{\prime}\right|-2$, and consequently, $P_{z}\left(Y^{\prime}\right) \leq a^{\left|Y^{\prime}\right|-2}(a-1)^{2}$.

Proof. Suppose towards a contradiction that $Y \in\left(\begin{array}{c}{[n]} \\ s-t+1\end{array}\right)$ satisfies $S(Y) \geq a\left(\begin{array}{c}s-t+1 \\ 2\end{array}\right)$ but for all $Y \subseteq Y^{\prime} \subseteq[n]$ such that $s-t+1 \leq\left|Y^{\prime}\right| \leq s-1$, there is $z \in[n] \backslash Y^{\prime}$ with $S_{z}\left(Y^{\prime}\right)>a\left|Y^{\prime}\right|-2$. Apply this fact with $Y^{\prime}=Y$ to choose $z_{1} \in[n] \backslash Y$ such that $S_{z_{1}}(Y)>a|Y|-2$. Then inductively define a sequence $z_{2}, \ldots, z_{t-1}$ so that for each $1 \leq i \leq t-2, S_{z_{i+1}}\left(Y \cup\left\{z_{1}, \ldots, z_{i}\right\}\right) \geq a(s-t+1+i)-1$ (to define $z_{i+1}$, apply the fact with $Y^{\prime}=Y \cup\left\{z_{1}, \ldots, z_{i}\right\}$ ). Then $\left|Y \cup\left\{z_{1}, \ldots, z_{t-1}\right\}\right|=s$ and

$$
\begin{aligned}
S\left(Y \cup\left\{z_{1}, \ldots, z_{t-1}\right\}\right) & \geq S(Y)+S_{z_{1}}(Y)+S_{z_{2}}\left(Y \cup\left\{z_{1}\right\}\right)+\ldots+S_{z_{t-1}}\left(Y \cup\left\{z_{1}, \ldots, z_{t-2}\right\}\right) \\
& \geq a\left(\begin{array}{c}
s-t+1 \\
2
\end{array}\right)+a(s-t+1)-1+\ldots+a(s-1)-1 \\
& =a\left(\begin{array}{l}
s \\
2
\end{array}\right)-(t-1)>a\left(\begin{array}{l}
s \\
2
\end{array}\right)-t,
\end{aligned}
$$

contradicting that $G \in F(n, s, q)$. Therefore there is $Y \subseteq Y^{\prime} \subseteq[n]$ such that $s-t+1 \leq\left|Y^{\prime}\right| \leq s-1$ and for all $z \in[n] \backslash Y^{\prime}, S_{z}\left(Y^{\prime}\right) \leq a\left|Y^{\prime}\right|-2$. By Lemma 2, this implies $P_{z}\left(Y^{\prime}\right) \leq a^{\left|Y^{\prime}\right|-2}(a-1)^{2}$.

Lemma 5. Suppose $s, q, a, t$ are integers satisfying the hypotheses of case (b) of Theorem 4. Then there are constants $C>1$ and $0<\alpha<1$ such that for all $n \geq 1$ the following holds. Suppose $G \in F(n, s, q)$ and $k(G)$ is the maximal number of pairwise disjoint elements of $\left\{Y \in\left(\begin{array}{c}{[n]} \\ s-t+1\end{array}\right)\right.$ : $\left.S(G[Y]) \geq a\left(\begin{array}{c}s-t+1 \\ 2\end{array}\right)\right\}$. Then

$$
P(G) \leq C^{k(G)} \alpha^{k(G) n} \operatorname{ex}_{\Pi}(n, s, q)
$$

Proof. Set $\alpha=\left(\frac{a-1}{a}\right)^{\frac{1}{2 t(s-t)}}$. Choose $C \geq q^{\left(\begin{array}{c}s-1 \\ 2\end{array}\right)}$ sufficiently large so that $\operatorname{ex}_{\Pi}(n, s, q) \leq C \alpha^{n^{2}}$ holds for all $1 \leq n \leq s^{3}$. We proceed by induction on $n$. If $1 \leq n \leq s^{3}$ and $G \in F(n, s, q)$, then (8) is clearly true of $k(G)=0$. If $k(G) \geq 1$, then by choice of $C$ and since $k(G) \leq n$ and $\alpha<1$,

$$
P(G) \leq \operatorname{ex}_{\Pi}(n, s, q) \leq C \alpha^{n^{2}} \leq C \alpha^{k(G) n} \leq C^{k(G)} \alpha^{k(G) n} \operatorname{ex}_{\Pi}(n, s, q) .
$$


Now let $n>s^{3}$ and suppose by induction (8) holds for all $G^{\prime} \in F\left(n^{\prime}, s, q\right)$ where $1 \leq n^{\prime}<n$. If $G \in F(n, s, q)$, then (8) is clearly true if $k(G)=0$. If $k(G)>0$, let $Y_{1}, \ldots, Y_{k}$ be a maximal set of pairwise disjoint elements in $\left\{Y \in\left(\begin{array}{c}{[n]} \\ s-t+1\end{array}\right): S(G[Y]) \geq a\left(\begin{array}{c}s-t+1 \\ 2\end{array}\right)\right\}$. Apply Lemma 4 to find $Y^{\prime}$ such that $Y_{1} \subseteq Y^{\prime} \subseteq[n], s-t+1 \leq\left|Y^{\prime}\right| \leq s-1$, and for all $z \in[n] \backslash Y^{\prime}, P_{z}\left(Y^{\prime}\right) \leq a^{\left|Y^{\prime}\right|-2}(a-1)^{2}$. Let $\left|Y^{\prime}\right|=y$. Then note

$$
P\left(Y^{\prime},[n] \backslash Y^{\prime}\right)=\prod_{z \in[n] \backslash Y^{\prime}} P_{z}\left(Y^{\prime}\right) \leq\left(a^{y-2}(a-1)^{2}\right)^{n-y} .
$$

Observe that $G\left[[n] \backslash Y^{\prime}\right]$ is isomorphic to some $H \in F(n-y, s, q)$. Since $Y^{\prime}$ can intersect at most $t-2$ other $Y_{i}$, and since $Y_{1}, \ldots, Y_{k}$ was maximal, we must have $k(H)+1 \leq k(G) \leq k(H)+t-1$. By our induction hypothesis,

$$
P\left([n] \backslash Y^{\prime}\right)=P(H) \leq C^{k(H)} \alpha^{k(H)(n-y)} \operatorname{ex}_{\Pi}(n-y, s, q) .
$$

Since $\mu(G) \leq q$ and $y \leq s-1$, and by our choice of $C, P\left(Y^{\prime}\right) \leq q^{\left(\begin{array}{c}y \\ 2\end{array}\right)} \leq C$. Combining this with (9), (10) and the fact that $\mu(H) \leq \mu(G)$ we obtain that

$$
\begin{aligned}
P(G)=P\left([n] \backslash Y^{\prime}\right) P\left(Y^{\prime},[n] \backslash Y^{\prime}\right) P\left(Y^{\prime}\right) & \leq C^{k(H)} \alpha^{k(H)(n-y)} \operatorname{ex}_{\Pi}(n-y, s, q)\left(a^{y-2}(a-1)^{2}\right)^{n-y} C \\
& =C^{k(H)+1} \alpha^{k(H)(n-y)} \operatorname{ex}_{\Pi}(n-y, s, q)\left(a^{y-2}(a-1)^{2}\right)^{n-y} .
\end{aligned}
$$

Plugging in the upper bound for $\operatorname{ex}_{\Pi}(n-y, s, q)$ from Claim 1 yields that $P(G)$ is at most

$$
C^{k(H)+1} \alpha^{k(H)(n-y)} \operatorname{ex}_{\Pi}(n, s, q)(a-1)^{-\left(\begin{array}{c}
y \\
2
\end{array}\right)}\left(\frac{a-1}{a}\right)^{\frac{n-y}{s-t}} \leq C^{k(H)+1} \alpha^{k(H)(n-y)+2 t(n-y)} \operatorname{ex}_{\Pi}(n, s, q),
$$

where the last inequality is because $(a-1)^{-\left(\begin{array}{c}y \\ 2\end{array}\right)}<1$ and by definition of $\alpha,\left(\frac{a-1}{a}\right)^{1 /(s-t)}=\alpha^{2 t}$. We claim that the following holds.

$$
k(H)(n-y)+2 t(n-y) \geq(k(H)+t-1) n .
$$

Rearranging this, we see (12) is equivalent to $y k(H) \leq t n+n-2 t y$. Since $2 \leq t \leq s / 2$ and $y \leq s-1, t n+n-2 t y \geq 3 n-s(s-1)$, so it suffices to show $y k(H) \leq 3 n-s(s-1)$. By definition, $k(H) \leq \frac{n-y}{s-t+1}$ so $y k(H) \leq \frac{y(n-y)}{s-t+1}$. Combining this with the facts that $s-t+1 \leq y \leq s-1$ and $s / 2<s-t+1$ yields

$$
y k(H) \leq \frac{(s-1)(n-(s-t+1))}{s-t+1}=n\left(\frac{s-1}{s-t+1}\right)-s+1<2 n\left(\frac{s-1}{s}\right)-s+1 .
$$

Thus it suffices to check $2 n\left(\frac{s-1}{s}\right)-s+1 \leq 3 n-s(s-1)$. This is equivalent to $(s-1)^{2} \leq n\left(\frac{s+2}{s}\right)$, which holds because $n \geq s^{3}$. This finishes the verification of (12). Combining (11), (12), and the fact that $k(H)+1 \leq k(G) \leq k(H)+t-1$ yields

$$
P(G) \leq C^{k(H)+1} \alpha^{(k(H)+t-1) n} \operatorname{ex}_{\Pi}(n, s, q) \leq C^{k(G)} \alpha^{k(G) n} \operatorname{ex}_{\Pi}(n, s, q) .
$$


Proof of Theorem 4(b) (Extremal). Set $s^{\prime}=s-t+1$ and $q^{\prime}=a\left(\begin{array}{l}s^{\prime} \\ 2\end{array}\right)-1$. Fix $n \geq s$. By Lemma 3 and definition of $s^{\prime}, \mathbb{T}_{s-t, a}(n)=\mathbb{T}_{s^{\prime}-1, a}(n) \subseteq \mathcal{P}(n, s, q)$ and

$$
\operatorname{ex}_{\Pi}(n, s, q)=\operatorname{ex}_{\Pi}\left(n, s^{\prime}, q^{\prime}\right)=(a-1)^{\left(\begin{array}{c}
n \\
2
\end{array}\right)}\left(\frac{a}{a-1}\right)^{t_{s^{\prime}-1}(n)}
$$

where the last equality is by Theorem 4(a) (Extremal) applied to $s^{\prime}$ and $q^{\prime}$. By definition, we have $\operatorname{ex}_{\Pi}(s, q)=(a-1)\left(\frac{a}{a-1}\right)^{1-\frac{1}{s^{\prime}-1}}$. We have left to show that $\mathcal{P}(n, s, q) \subseteq \mathbb{T}_{s^{\prime}-1, a}(n)$ holds for large $n$. Assume $n$ is sufficiently large and $C$ and $\alpha$ are as in Lemma 5. Note $\operatorname{ex}_{\Pi}(n, s, q)=\operatorname{ex}_{\Pi}\left(n, s^{\prime}, q^{\prime}\right)$ implies $\mathcal{P}(n, s, q) \cap F\left(n, s^{\prime}, q^{\prime}\right) \subseteq \mathcal{P}\left(n, s^{\prime}, q^{\prime}\right)=\mathbb{T}_{s^{\prime}-1, a}(n)$, where the equality is by Theorem 4 (a) (Extremal). So it suffices to show $\mathcal{P}(n, s, q) \subseteq F\left(n, s^{\prime}, q^{\prime}\right)$. Suppose towards a contradiction that there exists $G=([n], w) \in \mathcal{P}(n, s, q) \backslash F\left(n, s^{\prime}, q^{\prime}\right)$. Then in the notation of Lemma 5, $k(G) \geq 1$. Combining this with Lemma 5, we have

$$
P(G) \leq C^{k(G)} \alpha^{k(G) n} \operatorname{ex}_{\Pi}(n, s, q)=\left(C \alpha^{n}\right)^{k(G)} \operatorname{ex}_{\Pi}(n, s, q)<\operatorname{ex}_{\Pi}(n, s, q),
$$

where the last inequality is because $n$ is large, $\alpha<1$, and $k(G) \geq 1$. But now $P(G)<\operatorname{ex}_{\Pi}(n, s, q)$ contradicts that $G \in \mathcal{P}(n, s, q)$.

\section{Stability}

In this section we prove the product-stability results for Theorems 3 and 4 (a). We will use the fact that for any $(s, q)$-graph $G, \mu(G) \leq q$. If $G=(V, w)$ and $a \in \mathbb{N}$, let $E_{a}(G)=\left\{x y \in\left(\begin{array}{c}V \\ 2\end{array}\right): w(x y)=a\right\}$ and $e_{a}(G)=\left|E_{a}(G)\right|$. In the following notation, $p$ stands for "plus" and $m$ stands for "minus."

$$
p_{a}(G)=\left|\left\{x y \in\left(\begin{array}{l}
V \\
2
\end{array}\right): w(x y)>a\right\}\right| \quad \text { and } \quad m_{a}(G)=\left|\left\{x y \in\left(\begin{array}{l}
V \\
2
\end{array}\right): w(x y)<a\right\}\right| .
$$

Lemma 6. Let $s \geq 2, q \geq\left(\begin{array}{l}s \\ 2\end{array}\right)$ and $a>0$. Suppose there exist $0<\alpha<1$ and $C>1$ such that for all $n \geq s$, every $G \in F(n, s, q)$ satisfies

$$
P(G) \leq \operatorname{ex}_{\Pi}(n, s, q) q^{C n} \alpha^{p_{a}(G)} .
$$

Then for all $\delta>0$ there are $\epsilon, M>0$ such that for all $n>M$ the following holds. If $G \in F(n, s, q)$ and $P(G) \geq \operatorname{ex}_{\Pi}(n, s, q)^{1-\epsilon}$ then $p_{a}(G) \leq \delta n^{2}$.

Proof. Fix $\delta>0$. Choose $\epsilon>0$ so that $\frac{2 \epsilon \log q}{\log (1 / \alpha)}=\delta$. Choose $M \geq s$ sufficiently large so that $n \geq M$ implies $\left(\epsilon n^{2}+C n\right) \log q \leq 2 \epsilon \log q n^{2}$. Let $n>M$ and $G \in F(n, s, q)$ be such that $P(G) \geq \operatorname{ex}_{\Pi}(n, s, q)^{1-\epsilon}$. Our assumptions imply

$$
\operatorname{ex}_{\Pi}(n, s, q)^{1-\epsilon} \leq P(G) \leq \operatorname{ex}_{\Pi}(n, s, q) q^{C n} \alpha^{p_{a}(G)} .
$$

Rearranging $\operatorname{ex}_{\Pi}(n, s, q)^{1-\epsilon} \leq \operatorname{ex}_{\Pi}(n, s, q) q^{C n} \alpha^{p_{a}(G)}$ yields $\left(\frac{1}{\alpha}\right)^{p_{a}(G)} \leq \operatorname{ex}_{\Pi}(n, s, q)^{\epsilon} q^{C n} \leq q^{\epsilon n^{2}+C n}$, where the second inequality is because $\operatorname{ex}_{\Pi}(n, s, q) \leq q^{n^{2}}$. Taking logs of both sides, we obtain

$$
p_{a}(G) \log (1 / \alpha) \leq\left(\epsilon n^{2}+C n\right) \log q \leq 2 \epsilon n^{2} \log q,
$$

where the second inequality is by assumption on $n$. Dividing both sides by $\log (1 / \alpha)$ and applying the definition of $\epsilon$ yields $p_{a}(G) \leq \frac{2 \epsilon n^{2} \log q}{\log (1 / \alpha)}=\delta n^{2}$. 
We now prove the key lemma for this section.

Lemma 7. Let $s, q, b, a$ be integers satisfying $s \geq 2$ and either

(i) $a \geq 1,0 \leq b \leq s-2$, and $q=a\left(\begin{array}{l}s \\ 2\end{array}\right)+b$ or

(ii) $a \geq 2, b=0$, and $q=a\left(\begin{array}{c}s \\ 2\end{array}\right)-1$.

Then there exist $0<\alpha<1$ and $C>1$ such that for all $n \geq s$ and all $G \in F(n, s, q)$,

$$
P(G) \leq \operatorname{ex}_{\Pi}(n, s, q) q^{C n} \alpha^{p_{a}(G)} .
$$

Proof. We prove this by induction on $s \geq 2$, and for each fixed $s$, by induction on $n$. Let $s \geq 2$ and $q, b, a$ be as in (i) or (ii) above. Set

$$
\xi= \begin{cases}0 & \text { if case (i) holds } \\ 1 & \text { if case (ii) holds }\end{cases}
$$

Suppose first $s=2$. Set $\alpha=1 / 2$ and $C=2$. Since $G$ is an $(n, 2, a-\xi)$-graph, $p_{a}(G)=0$. Therefore for all $n \geq 2$,

$$
P(G) \leq \operatorname{ex}_{\Pi}(n, s, q) \leq \operatorname{ex}_{\Pi}(n, s, q) q^{C n}=\operatorname{ex}_{\Pi}(n, s, q) q^{C n} \alpha^{p_{a}(G)} .
$$

Assume now $s>2$. Let $\mathcal{I}$ be the set of $\left(s^{\prime}, q^{\prime}, b^{\prime}\right) \in \mathbb{N}^{3}$ such that $2 \leq s^{\prime}<s$ and $s^{\prime}, q^{\prime}, b^{\prime}, a$ satisfy (i) or (ii). Observe that $\mathcal{I}$ is finite. Suppose by induction on $s$ that $\left(s^{\prime}, q^{\prime}, b^{\prime}\right) \in \mathcal{I}$ implies there are $0<\alpha\left(s^{\prime}, q^{\prime}, b^{\prime}\right)<1$ and $C\left(s^{\prime}, q^{\prime}, b^{\prime}\right)>1$ such that for all $n^{\prime} \geq s^{\prime}$ and $G^{\prime} \in F\left(n^{\prime}, s^{\prime}, q^{\prime}\right)$, $P(G) \leq \operatorname{ex}_{\Pi}\left(n, s^{\prime}, q^{\prime}\right) q^{C\left(s^{\prime}, q^{\prime}, b^{\prime}\right) n} \alpha\left(s^{\prime}, q^{\prime}, b^{\prime}\right)^{p_{a}(G)}$. Set

$$
\alpha=\max \left(\left\{q^{-1},\left(\frac{a^{s-2}(a-\xi)-1}{a^{s-2}(a-\xi)}\right)^{\frac{1}{s-2}},\left(\frac{a-1}{a}\right)^{\frac{1}{s-2}}\right\} \cup\left\{\alpha\left(s^{\prime}, q^{\prime}, b^{\prime}\right):\left(s^{\prime}, q^{\prime}, b^{\prime}\right) \in \mathcal{I}\right\}\right) .
$$

Observe $0<\alpha<1$. Choose $C \geq\left(\begin{array}{c}s-1 \\ 2\end{array}\right)$ sufficiently large so that for all $n \leq s$

$$
q^{\left(\begin{array}{c}
n \\
2
\end{array}\right)} \leq q^{C n}(a-\xi)^{\left(\begin{array}{c}
n \\
2
\end{array}\right)}\left(\frac{a}{a-\xi}\right)^{t_{s-1}(n)} \alpha^{\left(\begin{array}{c}
n \\
2
\end{array}\right)}
$$

and so that for all $\left(s^{\prime}, q^{\prime}, b^{\prime}\right) \in \mathcal{I}, C\left(s^{\prime}, q^{\prime}, b^{\prime}\right) \leq C / 2$ and $\left(\frac{a+1}{a}\right)^{(s-3) /(s-2)} \leq q^{C / 2}$. Given $G \in$ $F(n, s, q)$, set

$$
\Theta(G)=\left\{Y \subseteq\left(\begin{array}{c}
{[n]} \\
s-1
\end{array}\right): S(Y) \geq a\left(\begin{array}{c}
s-1 \\
2
\end{array}\right)+(1-\xi) b\right\}
$$

and let $A(n, s, q)=\{G \in F(n, s, q): \Theta(G) \neq \emptyset\}$. We show the following holds for all $n \geq 1$ and $G \in F(n, s, q)$ by induction on $n$.

$$
P(G) \leq q^{C n}(a-\xi)^{\left(\begin{array}{l}
n \\
2
\end{array}\right)}\left(\frac{a}{a-\xi}\right)^{t_{s-1}(n)} \alpha^{p_{a}(G)} .
$$

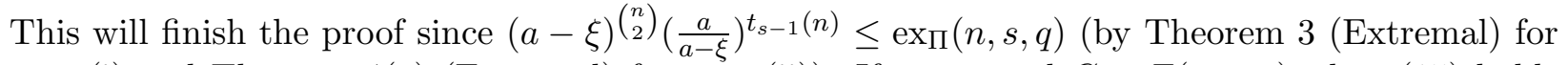
case (i) and Theorem 4(a) (Extremal) for case (ii)). If $n \leq s$ and $G \in F(n, s, q)$, then (15) holds

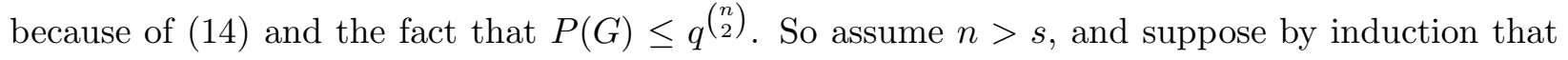


(15) holds for all $s \leq n^{\prime}<n$ and $G^{\prime} \in F\left(n^{\prime}, s, q\right)$. Let $G=([n], w) \in F(n, s, q)$. Suppose first that $G \in A(n, s, q)$. Choose $Y \in \Theta(G)$ and set $R=[n] \backslash Y$. Given $z \in R$, note that

$$
a\left(\begin{array}{c}
s-1 \\
2
\end{array}\right)+(1-\xi) b+S_{z}(Y) \leq S(Y)+S_{z}(Y)=S(Y \cup\{z\}) \leq a\left(\begin{array}{l}
s \\
2
\end{array}\right)+(1-\xi) b-\xi
$$

and therefore $S_{z}(Y) \leq a(s-1)-\xi$. Then for all $z \in R$, Lemma 2 implies $P_{z}(Y) \leq a^{s-2}(a-\xi)$, with equality only if $\{w(y z): y \in Y\}$ consists of $s-1-\xi$ elements equal to $a$ and $\xi$ elements equal to $a-1$. Let $R_{1}=\{z \in R: \exists y \in Y, w(z y)>a\}$ and $R_{2}=R \backslash R_{1}$. Then $z \in R_{1}$ implies $P_{z}(Y)<a^{s-2}(a-\xi)$, so $P_{z}(Y) \leq a^{s-2}(a-\xi)-1$. Let $k=\left|R_{1}\right|$. Observe that $G[R]$ is isomorphic to an element of $F\left(n^{\prime}, s, q\right)$, where $n^{\prime}=n-|R| \geq 1$. By induction (on $n$ ) and these observations we have that the following holds, where $p_{a}(R)=p_{a}(G[R])$.

$$
\begin{aligned}
P(G) & =P(R) P(Y) \prod_{z \in R_{1}} P_{z}(Y) \prod_{z \in R_{2}} P_{z}(Y) \\
& \leq q^{C(n-s+1)}(a-\xi)^{\left(\begin{array}{c}
n-s+1 \\
2
\end{array}\right)}\left(\frac{a}{a-\xi}\right)^{t_{s-1}(n-s+1)} \alpha^{p_{a}(R)} q^{\left({ }^{s-1}\right)}\left(a^{s-2}(a-\xi)-1\right)^{k}\left(a^{s-2}(a-\xi)\right)^{n-s+1-k} \\
& \leq q^{C(n-s+2)}(a-\xi)^{\left(\begin{array}{c}
n-s+1 \\
2
\end{array}\right)}\left(\frac{a}{a-\xi}\right)^{t_{s-1}(n-s+1)} \alpha^{p_{a}(R)}\left(a^{s-2}(a-\xi)-1\right)^{k}\left(a^{s-2}(a-\xi)\right)^{n-s+1-k}
\end{aligned}
$$

where the second inequality is because $\left(\begin{array}{c}s-1 \\ 2\end{array}\right) \leq C$. Since $\alpha \geq\left(\frac{a^{s-2}(a-\xi)-1}{a^{s-2}(a-\xi)}\right)^{1 /(s-2)}$, this is at most

$$
q^{C(n-s+2)}(a-\xi)^{\left({ }^{n-s+1}\right)}\left(\frac{a}{a-\xi}\right)^{t_{s-1}(n-s+1)} \alpha^{p_{a}(R)+k(s-1)}\left(a^{s-2}(a-\xi)\right)^{n-s+1} .
$$

Because $C(n-s+2) \leq C n-\left(\begin{array}{c}s-1 \\ 2\end{array}\right)$ and $q^{-1} \leq \alpha$, we have $q^{C(n-s+2)} \leq q^{C n} \alpha^{\left(\begin{array}{c}s-1 \\ 2\end{array}\right)}$. Combining this with the fact that $p_{a}(G) \leq p_{a}(R)+k(s-1)+\left(\begin{array}{c}s-1 \\ 2\end{array}\right)$ implies that (16) is at most

$$
\begin{aligned}
& q^{C n}(a-\xi)^{\left(\begin{array}{c}
n-s+1 \\
2
\end{array}\right)}\left(\frac{a}{a-\xi}\right)^{t_{s-1}(n-s+1)} \alpha^{p_{a}(R)+k(s-1)+\left(\begin{array}{c}
s-1 \\
2
\end{array}\right)}\left(a^{s-2}(a-\xi)\right)^{n-s+1} \\
= & q^{C n}(a-\xi)^{\left(\begin{array}{c}
n-s+1 \\
2
\end{array}\right)+(s-1)(n-s+1)}\left(\frac{a}{a-\xi}\right)^{t_{s-1}(n-s+1)+(s-2)(n-s+1)} \alpha^{p_{a}(R)+k(s-1)+\left(\begin{array}{c}
s-1 \\
2
\end{array}\right)} \\
\leq & q^{C n}(a-\xi)^{\left(\begin{array}{c}
n \\
2
\end{array}\right)}\left(\frac{a}{a-\xi}\right)^{t_{s-1}(n)} \alpha^{p_{a}(G)} .
\end{aligned}
$$

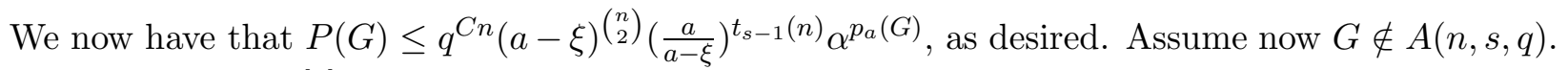
Then for all $Y \in\left(\begin{array}{c}{[n]} \\ s-1\end{array}\right), S(Y) \leq a\left(\begin{array}{c}s-1 \\ 2\end{array}\right)+(1-\xi) b-1$. Thus $G$ is an $\left(n, s^{\prime}, q^{\prime}\right)$-graph where $s^{\prime}=s-1$ and $q^{\prime}=a\left(\begin{array}{c}s-1 \\ 2\end{array}\right)+(1-\xi) b-1$. Suppose $a=1, \xi=0$, and $b=0$. Then $q^{\prime}=\left(\begin{array}{c}s^{\prime} \\ 2\end{array}\right)-1$ and any $\left(n, s^{\prime}, q^{\prime}\right)$-graph must contain an edge of multiplicity 0 . This implies $P(G)=0$ and (15) holds. We have the following three cases remaining, where $b^{\prime}=\max \{b-1,0\}$.

1. $\xi=0, b=0$, and $a \geq 2$. In this case $q^{\prime}=a\left(\begin{array}{c}s^{\prime} \\ 2\end{array}\right)-1$ and $b^{\prime}=0$.

2. $\xi=1, b=0$, and $a \geq 2$. In this case $q^{\prime}=a\left(\begin{array}{c}s^{\prime} \\ 2\end{array}\right)-1$ and $b^{\prime}=0$.

3. $\xi=0,1 \leq b \leq s-2$, and $a \geq 1$. In this case $q^{\prime}=a\left(\begin{array}{c}s^{\prime} \\ 2\end{array}\right)+b^{\prime}$ and $0 \leq b^{\prime} \leq s^{\prime}-2$. 
It is clear that in all three of these cases, $\left(s^{\prime}, q^{\prime}, b^{\prime}\right) \in \mathcal{I}$, so by our induction hypothesis (on $s$ ), there are $\alpha^{\prime}=\alpha\left(s^{\prime}, q^{\prime}, b^{\prime}\right) \leq \alpha$ and $C^{\prime}=C\left(s^{\prime}, q^{\prime}, b\right)$ such that

$$
P(G) \leq \operatorname{ex}_{\Pi}\left(n, s^{\prime}, q^{\prime}\right)\left(q^{\prime}\right)^{C^{\prime} n}\left(\alpha^{\prime}\right)^{p_{a}(G)} \leq \operatorname{ex}_{\Pi}\left(n, s^{\prime}, q^{\prime}\right) q^{C^{\prime} n} \alpha^{p_{a}(G)},
$$

where the inequality is because $q^{\prime} \leq q$ and $\alpha^{\prime} \leq \alpha$. By Theorem 4(a) (Extremal) if cases 1 or 2 hold, and by Theorem 3 (Extremal) if case 3 holds, we have the following.

$$
\operatorname{ex}_{\Pi}\left(n, s^{\prime}, q^{\prime}\right) \leq(a-\xi)^{\left(\begin{array}{c}
n \\
2
\end{array}\right)}\left(\frac{a}{a-\xi}\right)^{t_{s^{\prime}-1}(n)}\left(\frac{a+1}{a}\right)^{\left\lfloor\frac{b^{\prime}}{b^{\prime}+1} n\right\rfloor} \leq(a-\xi)^{\left(\begin{array}{l}
n \\
2
\end{array}\right)}\left(\frac{a}{a-\xi}\right)^{t_{s-1}(n)}\left(\frac{a+1}{a}\right)^{\frac{s-3}{s-2} n},
$$

where the last inequality is because $t_{s^{\prime}-1}(n) \leq t_{s-1}(n)$ and $\left\lfloor\frac{b^{\prime}}{b^{\prime}+1} n\right\rfloor \leq \frac{b^{\prime}}{b^{\prime}+1} n \leq \frac{s-3}{s-2} n$. By choice of

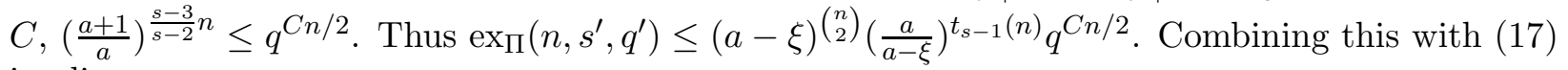
implies

$$
P(G) \leq(a-\xi)^{\left(\begin{array}{c}
n \\
2
\end{array}\right)}\left(\frac{a}{a-\xi}\right)^{t_{s-1}(n)} q^{C n / 2} q^{C^{\prime} n} \alpha^{p_{a}(G)} \leq(a-\xi)^{\left(\begin{array}{c}
n \\
2
\end{array}\right)}\left(\frac{a}{a-\xi}\right)^{t_{s-1}(n)} q^{C n} \alpha^{p_{a}(G)},
$$

where the last inequality is because $C^{\prime} \leq C / 2$. Thus (15) holds.

Proof of Theorem 3 (Stability). Let $s \geq 2, a \geq 1$, and $q=a\left(\begin{array}{c}s \\ 2\end{array}\right)+b$ for some $0 \leq b \leq s-2$. Fix $\delta>0$. Given $G \in F(n, s, q)$, let $p_{G}=p_{a}(G)$ and $m_{G}=m_{a}(G)$. Note that if $G \in F(n, s, q)$, then $\left|\Delta\left(G, \mathbb{U}_{a}(n)\right)\right|=m_{G}+p_{G}$. Suppose first $a=1$, so $m_{G}=0$. Combining Lemma 7 with Lemma 6 implies there are $\epsilon_{1}$ and $M_{1}$ such that if $n>M_{1}$ and $G \in F(n, s, q)$ satisfies $P(G) \geq \operatorname{ex}_{\Pi}(n, s, q)^{1-\epsilon_{1}}$, then $\left|\Delta\left(G, \mathbb{U}_{a}(n)\right)\right|=p_{G} \leq \delta n^{2}$. Assume now $a>1$. Combining Lemma 7 with Lemma 6 implies there are $\epsilon_{1}$ and $M_{1}$ such that if $n>M_{1}$ and $G \in F(n, s, q)$ satisfies $P(G) \geq \operatorname{ex}_{\Pi}(n, s, q)^{1-\epsilon_{1}}$, then $p_{G} \leq \delta^{\prime} n^{2}$, where

$$
\delta^{\prime}=\min \left\{\frac{\delta}{2}, \frac{\delta \log (a /(a-1))}{4 \log q}\right\} .
$$

Set $\epsilon=\min \left\{\epsilon_{1}, \frac{\delta \log (a /(a-1))}{4 \log q}\right\}$. Suppose $n>M_{1}$ and $G \in F(n, s, q)$ satisfies $P(G) \geq \operatorname{ex}_{\Pi}(n, s, q)^{1-\epsilon}$. Our assumptions imply $p_{G} \leq \delta^{\prime} n^{2} \leq \delta n^{2} / 2$. Observe that by definition of $p_{G}$ and $m_{G}$,

$$
P(G) \leq a^{\left(\begin{array}{c}
n \\
2
\end{array}\right)-m_{G}}(a-1)^{m_{G}} q^{p_{G}}=a^{\left(\begin{array}{c}
n \\
2
\end{array}\right)}\left(\frac{a-1}{a}\right)^{m_{G}} q^{p_{G}} .
$$

By Theorem $3\left(\right.$ a) (Extremal), $\operatorname{ex}_{\Pi}(n, s, q) \geq a^{\left(\begin{array}{c}n \\ 2\end{array}\right)}$. Therefore $P(G) \geq \operatorname{ex}_{\Pi}(n, s, q)^{1-\epsilon} \geq a^{\left(\begin{array}{c}n \\ 2\end{array}\right)(1-\epsilon)}$. Combining this with (18) yields

$$
a^{\left(\begin{array}{c}
n \\
2
\end{array}\right)(1-\epsilon)} \leq a^{\left(\begin{array}{c}
n \\
2
\end{array}\right)}\left(\frac{a-1}{a}\right)^{m_{G}} q^{p_{G}} .
$$

Rearranging this, we obtain

$$
\left(\frac{a}{a-1}\right)^{m_{G}} \leq a^{\epsilon\left(\begin{array}{c}
n \\
2
\end{array}\right)} q^{p_{G}} \leq q^{\epsilon\left(\begin{array}{c}
n \\
2
\end{array}\right)+p_{G}} \leq q^{\epsilon n^{2}+p_{G}} .
$$

Taking logs, dividing by $\log (a /(a-1))$, and applying our assumptions on $p_{G}$ and $\epsilon$ yields

$$
m_{G} \leq \frac{\epsilon n^{2} \log q}{\log (a /(a-1))}+\frac{p_{G} \log q}{\log (a /(a-1)} \leq \frac{\delta n^{2}}{4}+\frac{\delta n^{2}}{4}=\frac{\delta n^{2}}{2}
$$

Combining this with the fact that $p_{G} \leq \frac{\delta n^{2}}{2}$ we have that $\left|\Delta\left(G, \mathbb{U}_{a}(n)\right)\right| \leq \delta n^{2}$.

The following classical result gives structural information about $n$-vertex $K_{s}$-free graphs with close to $t_{s-1}(n)$ edges. 
Theorem 12 (Erdös-Simonovits [5, 16]). For all $\delta>0$ and $s \geq 2$, there is an $\epsilon>0$ such that every $K_{s}$-free graph with $n$ vertices and $t_{s-1}(n)-\epsilon n^{2}$ edges can be transformed into $T_{s-1}(n)$ by adding and removing at most $\delta n^{2}$ edges.

Proof of Theorem 4 (a) (Stability). Let $s \geq 2, a \geq 2$, and $q=a\left(\begin{array}{c}s \\ 2\end{array}\right)-1$. Fix $\delta>0$. Given $G \in F(n, s, q)$, let $p_{G}=p_{a}(G), m_{G}=m_{a-1}(G)$. Choose $M_{0}$ and $\mu$ such that $\mu<\delta / 2$ and so that Theorem 12 implies that any $K_{s}$-free graph with $n \geq M_{0}$ vertices and at least $(1-\mu) t_{s-1}(n)$ edges can be made into $T_{s-1}(n)$ by adding or removing at most $\frac{\delta n^{2}}{3}$ edges. Set

$$
A= \begin{cases}2 & \text { if } a=2 \\ \frac{a-1}{a-2} & \text { if } a>2\end{cases}
$$

Combining Lemma 7 with Lemma 6 implies there are $\epsilon_{1}, M_{1}$ so that if $n>M_{1}$ and $G \in F(n, s, q)$ satisfies $P(G) \geq \operatorname{ex}_{\Pi}(n, s, q)^{1-\epsilon_{1}}$, then $p_{G} \leq \delta^{\prime} n^{2}$, where

$$
\delta^{\prime}=\min \left\{\frac{\delta}{3}, \frac{\mu \log (a /(a-1))}{2 \log q}, \frac{\delta \log A}{6 \log q}\right\} .
$$

Let

$$
\epsilon=\min \left\{\epsilon_{1}, \frac{\delta \log A}{6 \log q}, \frac{\mu \log (a /(a-1))}{2 \log q}\right\} \quad \text { and } \quad M=\max \left\{M_{0}, M_{1}\right\} .
$$

Suppose now that $n>M$ and $G \in F(n, s, q)$ satisfies $P(G) \geq \operatorname{ex}_{\Pi}(n, s, q)^{1-\epsilon}$. By assumption, $p_{G} \leq \delta^{\prime} n^{2} \leq \frac{\delta n^{2}}{3}$. We now bound $m_{G}$. Note that if $a=2$ and $P(G) \neq 0$, then $m_{G}=0$. If $a>2$, observe that by definition of $p_{G}$ and $m_{G}$,

$$
P(G) \leq q^{p_{G}}(a-2)^{m_{G}} a^{e_{a}(G)}(a-1)^{e_{a-1}(G)} \leq q^{p_{G}}\left(\frac{a-2}{a-1}\right)^{m_{G}} a^{e_{a}(G)}(a-1)^{\left(\begin{array}{c}
n \\
2
\end{array}\right)-e_{a}(G)},
$$

where the last inequality is because $e_{a-1}(G)+m_{G} \leq\left(\begin{array}{c}n \\ 2\end{array}\right)-e_{a}(G)$. Note that Turán's theorem and the fact that $G$ is an $(n, s, q)$-graph implies that $e_{a}(G) \leq t_{s-1}(n)$, so

$$
a^{e_{a}(G)}(a-1)^{\left(\begin{array}{l}
n \\
2
\end{array}\right)-e_{a}(G)} \leq a^{t_{s-1}(n)}(a-1)^{\left(\begin{array}{l}
n \\
2
\end{array}\right)-t_{s-1}(n)}=\operatorname{ex}_{\Pi}(n, s, q),
$$

where the last equality is from Theorem 4(a) (Extremal). Combining this with (20) yields

$$
\operatorname{ex}_{\Pi}(n, s, q)^{1-\epsilon} \leq P(G) \leq q^{p_{G}}\left(\frac{a-2}{a-1}\right)^{m_{G}} \operatorname{ex}_{\Pi}(n, s, q) .
$$

Rearranging $\operatorname{ex}_{\Pi}(n, s, q)^{1-\epsilon} \leq q^{p_{G}}\left(\frac{a-2}{a-1}\right)^{m_{G}} \operatorname{ex}_{\Pi}(n, s, q)$ and using that $\operatorname{ex}_{\Pi}(n, s, q) \leq q^{n^{2}}$, we obtain

$$
A^{m_{G}}=\left(\frac{a-1}{a-2}\right)^{m_{G}} \leq q^{p_{G}} \operatorname{ex}_{\Pi}(n, s, q)^{\epsilon} \leq q^{p_{G}+\epsilon n^{2}} .
$$

Taking logs, dividing by $\log A$, and applying our assumptions on $p_{G}$ and $\epsilon$ we obtain $m_{G}<\delta n^{2} / 3$. Using (20) and $a^{t_{s-1}(n)}(a-1)^{\left(\begin{array}{c}n \\ 2\end{array}\right)-t_{s-1}(n)}=\operatorname{ex}_{\Pi}(n, s, q)$, we have

$$
\operatorname{ex}_{\Pi}(n, s, q)^{1-\epsilon} \leq P(G) \leq q^{p_{G}} a^{e_{a}(G)}(a-1)^{\left(\begin{array}{c}
n \\
2
\end{array}\right)-e_{a}(G)}=q^{p_{G}} \operatorname{ex}_{\Pi}(n, s, q)\left(\frac{a}{a-1}\right)^{e_{a}(G)-t_{s-1}(n)} .
$$

Rearranging this we obtain

$$
\left(\frac{a}{a-1}\right)^{t_{s-1}(n)-e_{a}(G)} \leq q^{p_{G}} \operatorname{ex}_{\Pi}(n, s, q)^{\epsilon} \leq q^{p_{G}+\epsilon n^{2}} .
$$


Taking logs, dividing by $\log (a /(a-1))$, and using the assumptions on $p_{G}$ and $\epsilon$ we obtain that

$$
t_{s-1}(n)-e_{a}(G) \leq \frac{p_{G} \log q}{\log (a /(a-1))}+\frac{\epsilon n^{2} \log q}{\log (a /(a-1))} \leq \frac{\mu n^{2}}{2}+\frac{\mu n^{2}}{2}=\mu n^{2} .
$$

Let $H$ be the graph with vertex set $[n]$ and edge set $E=E_{a}(G)$. Then $H$ is $K_{s}$-free, and has $e_{a}(G)$ many edges. Since $t_{s-1}(n)-e_{a}(G) \leq \mu n^{2}$, Theorem 12 implies that $H$ is $\frac{\delta}{3}$-close to some $H^{\prime}=T_{s-1}(n)$. Define $G^{\prime} \in F(n, s, q)$ so that $E_{a}\left(G^{\prime}\right)=E\left(H^{\prime}\right)$ and $E_{a-1}\left(G^{\prime}\right)=\left(\begin{array}{l}n \\ 2\end{array}\right) \backslash E_{a}\left(G^{\prime}\right)$. Then $G^{\prime} \in \mathbb{T}_{s-1, a}(n)$ and

$$
\Delta\left(G, G^{\prime}\right) \subseteq\left(E_{a}(G) \Delta E_{a}\left(G^{\prime}\right)\right) \cup \bigcup_{i \notin\{a, a-1\}} E_{i}(G)=\Delta\left(H, H^{\prime}\right) \cup \underset{i \notin\{a, a-1\}}{\bigcup} E_{i}(G) .
$$

This implies $\left|\Delta\left(G, G^{\prime}\right)\right| \leq\left|\Delta\left(H, H^{\prime}\right)\right|+p_{G}+m_{G} \leq \frac{\delta}{3} n^{2}+\frac{\delta}{3} n^{2}+\frac{\delta}{3} n^{2}=\delta n^{2}$.

\subsection{Proof of Theorem 4 (b) (Stability)}

In this subsection we prove Theorem 4(b) (Stability). We first prove two lemmas.

Lemma 8. Let $s \geq 4, a \geq 2$, and $q=a\left(\begin{array}{c}s \\ 2\end{array}\right)-t$ for some $2 \leq t \leq \frac{s}{2}$. For all $\lambda>0$ there are $M$ and $\epsilon>0$ such that the following holds. Suppose $n>M$ and $G \in F(n, s, q)$ satisfies $P(G)>\operatorname{ex}_{\Pi}(n, s, q)^{1-\epsilon}$. Then $k(G)<\lambda n$, where $k(G)$ is as defined in Lemma 5 .

Proof. Fix $\lambda>0$. Set $\eta=a^{\frac{s-t-1}{s-t}}(a-1)^{\frac{1}{s-t}}$ and choose $C$ and $\alpha$ as in Lemma 5 . Choose $\epsilon>0$ so that $\alpha^{\lambda / 2}=\eta^{-\epsilon}$. By Theorem 4(b) (Extremal), $\operatorname{ex}_{\Pi}(n, s, q)=\eta^{\left(\begin{array}{c}n \\ 2\end{array}\right)+o\left(n^{2}\right)}$. Assume $M$ sufficiently large so that for all $n \geq M$, (4) holds for all $G \in F(n, s, q)$, $\operatorname{ex}_{\Pi}(n, s, q)<\eta^{n^{2}}, C^{\lambda n} \leq \eta^{\epsilon n^{2}}$, and $C \alpha^{n}<1$. Fix $n \geq M$ and suppose towards a contradiction that $G \in F(n, s, q)$ satisfies $P(G)>\operatorname{ex}_{\Pi}(n, s, q)^{1-\epsilon}$ and $k(G) \geq \lambda n$. By Lemma 5 and the facts that $C \alpha^{n}<1$ and $k(G) \geq 1$, we obtain that

$$
P(G) \leq C^{k(G)} \alpha^{n k(G)} \operatorname{ex}_{\Pi}(n, s, q)=\left(C \alpha^{n}\right)^{k(G)} \operatorname{ex}_{\Pi}(n, s, q) \leq\left(C \alpha^{n}\right)^{\lambda n} \operatorname{ex}_{\Pi}(n, s, q) .
$$

By assumption on $n$ and definition of $\epsilon,\left(C \alpha^{n}\right)^{\epsilon n}=C^{\lambda n} \alpha^{\lambda n^{2}}=C^{\lambda n} \eta^{-2 \epsilon n^{2}} \leq \eta^{-\epsilon n^{2}}$. Thus

$$
P(G) \leq \eta^{-\epsilon n^{2}} \operatorname{ex}_{\Pi}(n, s, q)<\operatorname{ex}_{\Pi}(n, s, q)^{1-\epsilon},
$$

where the last inequality is because by assumption, $\operatorname{ex}_{\Pi}(n, s, q)<\eta^{n^{2}}$. But this contradicts our assumption that $P(G)>\operatorname{ex}_{\Pi}(n, s, q)^{1-\epsilon}$.

Given a multigraph $G=(V, w)$, let $\mathcal{H}(G, s, q)=\left\{Y \in\left(\begin{array}{l}V \\ s\end{array}\right): S(Y)>q\right\}$. Observe that $G$ is an $(s, q)$-graph if and only if $\mathcal{H}(G, s, q)=\emptyset$.

Lemma 9. Let $s, q, m \geq 2$ be integers. For all $0<\delta<1$, there is $0<\lambda<1$ and $N$ such that $n>N$ implies the following. If $G=([n], w)$ has $\mu(G) \leq m$ and $\mathcal{H}(G, s, q)$ contains strictly less than $\lceil\lambda n\rceil$ pairwise disjoint elements, then $G$ is $\delta$-close to an element in $F(n, s, q)$.

Proof. Fix $0<\delta<1$. Observe we can view any multigraph $G$ with $\mu(G) \leq m$ as an edgecolored graph with colors in $\{0, \ldots, m\}$. By Theorem 1, there is $\epsilon$ and $M$ such that if $n>M$ and $G=([n], w)$ has $\mu(G) \leq m$ and $\mathcal{H}(G, s, q) \leq \epsilon\left(\begin{array}{l}n \\ s\end{array}\right)$, then $G$ is $\delta$-close to an element of $F(n, s, q)$. Let $\lambda:=\epsilon / s$ and $N=\max \left\{M, \frac{s}{1-\lambda s}\right\}$. We claim this $\lambda$ and $N$ satisfy the desired conclusions. Suppose towards a contradiction that $n>M$ and $G=([n], w)$ has $\mu(G) \leq m, \mathcal{H}(n, s, q)$ contains 
strictly less than $\lceil\lambda n\rceil$ pairwise disjoint elements, but $G$ is $\delta$-far from every element in $F(n, s, q)$. Then $\mathcal{H}(G, s, q)>\epsilon\left(\begin{array}{l}n \\ s\end{array}\right)$ by choice of $M$ and $\lambda$. By our choice of $N,\lceil\lambda n\rceil s \leq(\lambda n+1) s \leq n$. Then Proposition 11.6 in [11] and our assumptions imply $|\mathcal{H}(G, s, q)| \leq(\lceil\lambda n\rceil-1)\left(\begin{array}{c}n-1 \\ s-1\end{array}\right)$. But now

$$
|\mathcal{H}(G, s, q)| \leq(\lceil\lambda n\rceil-1)\left(\begin{array}{c}
n-1 \\
s-1
\end{array}\right)<\lambda n\left(\begin{array}{c}
n-1 \\
s-1
\end{array}\right)=\left(\frac{\epsilon n}{s}\right)\left(\frac{s}{n}\right)\left(\begin{array}{c}
n \\
s
\end{array}\right)=\epsilon\left(\begin{array}{c}
n \\
s
\end{array}\right),
$$

a contradiction.

Proof of Theorem 4(b) (Stability). Let $s \geq 4, a \geq 2$, and $q=a\left(\begin{array}{l}s \\ 2\end{array}\right)-t$ for some $2 \leq t \leq \frac{s}{2}$. Fix $\delta>0$. Let $s^{\prime}=s-t+1$ and $q^{\prime}=a\left(\begin{array}{c}s^{\prime} \\ 2\end{array}\right)-1$. Note Theorem 4 (Extremal) implies that for sufficiently large $n, \mathcal{P}(n, s, q)=\mathbb{T}_{s^{\prime}-1, a}(n), \operatorname{ex}_{\Pi}\left(n, s^{\prime}, q^{\prime}\right)=\operatorname{ex}_{\Pi}(n, s, q)$, and $\operatorname{ex}_{\Pi}\left(s^{\prime}, q^{\prime}\right)=\operatorname{ex}_{\Pi}(s, q)=\eta$, where $\eta=(a-1)\left(\frac{a}{a-1}\right)^{\left(s^{\prime}-2\right) /\left(s^{\prime}-1\right)}$.

Apply Theorem 4 (a) (Stability) for $\left(s^{\prime}, q^{\prime}\right)$ to $\delta / 2$ to obtain $\epsilon_{0}$. By replacing $\epsilon_{0}$ if necessary, assume $\epsilon_{0}<4 \delta / \log \eta$. Set $\epsilon_{1}=\epsilon_{0} \log \eta /(8 \log q)$ and note $\epsilon_{1}<\delta / 2$. Apply Lemma 9 to $\epsilon_{1}$ and $m=q$ to obtain $\lambda$ such that for large $n$ the following holds. If $G=([n], w)$ has $\mu(G) \leq q$ and $\mathcal{H}\left(G, s^{\prime}, q^{\prime}\right)$ contains strictly less than $\lceil\lambda n\rceil$ pairwise disjoint elements, then $G$ is $\epsilon_{1}$-close to an element in $F\left(n, s^{\prime}, q^{\prime}\right)$. Finally, apply Lemma 8 for $s, q, t$ to $\lambda$ to obtain $\epsilon_{2}>0$.

Choose $M$ sufficiently large for the desired applications of Theorems 4(a) (Stability) and 4(b) (Extremal) and Lemmas 8 and 9. Set $\epsilon=\min \left\{\epsilon_{2}, \epsilon_{0} / 2\right\}$. Suppose $n>M$ and $G \in F(n, s, q)$ satisfies $P(G) \geq \operatorname{ex}_{\Pi}(n, s, q)^{1-\epsilon}$. Then Lemma 8 and our choice of $\epsilon$ implies $k(G)<\lambda n$. Observe that by the definitions of $s^{\prime}, q^{\prime}$,

$$
\left\{Y \in\left(\begin{array}{c}
{[n]} \\
s-t+1
\end{array}\right): S(Y) \geq a\left(\begin{array}{c}
s-t+1 \\
2
\end{array}\right)\right\}=\left\{Y \in\left(\begin{array}{c}
{[n]} \\
s^{\prime}
\end{array}\right): S(Y) \geq q^{\prime}+1\right\}=\mathcal{H}\left(G, s^{\prime}, q^{\prime}\right) .
$$

Thus $k(G)<\lambda n$ means $\mathcal{H}\left(G, s^{\prime}, q^{\prime}\right)$ contains strictly less than $\lceil\lambda n\rceil$ pairwise disjoint elements. Lemma 9 then implies $G$ is $\epsilon_{1}$-close to some $G^{\prime} \in F\left(n, s^{\prime}, q^{\prime}\right)$. Combining this with the definition of $\epsilon_{1}$ yields

$$
P\left(G^{\prime}\right) \geq P(G) q^{-\left|\Delta\left(G, G^{\prime}\right)\right|} \geq P(G) q^{-\epsilon_{1} n^{2}}=P(G) \eta^{-\epsilon_{0} n^{2} / 8} \geq \operatorname{ex}_{\Pi}(n, s, q)^{1-\epsilon} \eta^{-\left(\epsilon_{0} / 2\right)\left(\begin{array}{l}
n \\
2
\end{array}\right) .}
$$

By Proposition 1, $\operatorname{ex}_{\Pi}(n, s, q) \geq \operatorname{ex}_{\Pi}(s, q)\left(\begin{array}{l}n \\ 2\end{array}\right)=\eta^{\left(\begin{array}{c}n \\ 2\end{array}\right)}$. Combining this with (21) and the definition of $\epsilon$ yields

$$
P\left(G^{\prime}\right) \geq \operatorname{ex}_{\Pi}(n, s, q)^{1-\epsilon} \eta^{-\left(\epsilon_{0} / 2\right)\left(\begin{array}{l}
n \\
2
\end{array}\right)} \geq \operatorname{ex}_{\Pi}(n, s, q)^{1-\epsilon-\epsilon_{0} / 2} \geq \operatorname{ex}_{\Pi}(n, s, q)^{1-\epsilon_{0}} .
$$

Since $\operatorname{ex}_{\Pi}(n, s, q)=\operatorname{ex}_{\Pi}\left(n, s^{\prime}, q^{\prime}\right)$, (22) implies $P\left(G^{\prime}\right) \geq \operatorname{ex}_{\Pi}\left(n, s^{\prime}, q^{\prime}\right)^{1-\epsilon_{0}}$, so Theorem 4(a) (Stability) implies $G^{\prime}$ is $\delta / 2$-close to some $G^{\prime \prime} \in \mathbb{T}_{s^{\prime}-1, a}(n)=\mathbb{T}_{s-t, a}(n)$. Now we are done, since

$$
\left|\Delta\left(G, G^{\prime \prime}\right)\right| \leq\left|\Delta\left(G, G^{\prime}\right)\right|+\left|\Delta\left(G^{\prime}, G^{\prime \prime}\right)\right| \leq \epsilon_{1} n^{2}+\delta n^{2} / 2 \leq \delta n^{2} .
$$

\section{Extremal Result for $(n, 4,9)$-graphs}

In this section we prove Theorems [5. We first prove one of the inequalities needed for Theorem 5 ,

Lemma 10. For all $n \geq 4,2^{\operatorname{ex}\left(n,\left\{C_{3}, C_{4}\right\}\right)} \leq \operatorname{ex}_{\Pi}(n, 4,9)$. 
Proof. Fix $G=([n], E)$ an extremal $\left\{C_{3}, C_{4}\right\}$-free graph, and let $G^{\prime}=([n], w)$ where $w(x y)=2$ for all $x y \in E$ and $w(x y)=1$ for all $x y \in\left(\begin{array}{c}n \\ 2\end{array}\right) \backslash E$. Suppose $X \in\left(\begin{array}{c}{[n]} \\ 4\end{array}\right)$. Since $G$ is $\left\{C_{3}, C_{4}\right\}$-free, $\left|E \cap\left(\begin{array}{c}X \\ 2\end{array}\right)\right| \leq 3$. Thus $\left\{w(x y): x y \in\left(\begin{array}{c}X \\ 2\end{array}\right)\right\}$ contains at most 3 elements equal to 2 and the rest equal to 1 , so $S(X) \leq 9$. This shows $G^{\prime} \in F(n, 4,9)$. Thus $2^{|E|}=2^{\operatorname{ex}\left(n,\left\{C_{3}, C_{4}\right\}\right)}=P\left(G^{\prime}\right) \leq \operatorname{ex}_{\Pi}(n, 4,9)$.

To prove the reverse inequality, our strategy will be to show that if $G \in F(n, 4,9)$ has no edges of multiplicity larger than 2 , then $P(G) \leq 2^{\operatorname{ex}\left(n,\left\{C_{3}, C_{4}\right\}\right)}$ (Theorem 13). We will then show that all product extremal $(4,9)$-graphs have no edges of multiplicity larger than 2 (Theorem 14). Theorem 5 will then follow. We begin with a few definitions and lemmas.

Definition 9. Suppose $n \geq 1$. Set $F_{\leq 2}(n, 4,9)=\{G \in F(n, 4,9): \mu(G) \leq 2\}$ and

$$
D(n)=F_{\leq 2}(n, 4,9) \cap F(n, 3,5) .
$$

Lemma 11. For all $n \geq 4$, if $G=([n], w) \in D(n)$, then $P(G) \leq 2^{\operatorname{ex}\left(n,\left\{C_{3}, C_{4}\right\}\right)}$.

Proof. If $P(G)=0$ we are done, so assume $P(G)>0$. Let $H=([n], E)$ be the graph where $E=\left\{x y \in\left(\begin{array}{c}{[n]} \\ 2\end{array}\right): w(x y)=2\right\}$. Since $P(G)>0$ and $\mu(G) \leq 2, G$ contains all edges of multiplicity 1 or 2. Consequently, $P(G)=2^{|E|}$. Since $G \in F(n, 3,5), H$ is $C_{3}$-free and since $G \in F(n, 4,9), H$ is $C_{4}$-free, so $|E| \leq \operatorname{ex}\left(n,\left\{C_{3}, C_{4}\right\}\right)$. This shows $P(G)=2^{|E|} \leq 2^{\operatorname{ex}\left(n,\left\{C_{3}, C_{4}\right\}\right)}$.

The following lemma gives us useful information about elements of $F(n, 4,9) \backslash F(n, 3,5)$.

Lemma 12. Suppose $n \geq 4$ and $G=([n], w) \in F(n, 4,9)$ satisfies $P(G)>0$. If there is $X \in\left(\begin{array}{c}{[n]} \\ 3\end{array}\right)$ such that $S(X) \geq 6$, then $P(X) \leq 2^{3}$ and $w(x y)=1$ for all $x \in X$ and $y \in[n] \backslash X$. Consequently

$$
P(G)=P(X) P([n] \backslash X) \leq 2^{3} P([n] \backslash X) .
$$

Proof. Let $y \in[n] \backslash X$. Since $P(G)>0$, every edge in $G$ has multiplicity at least 1 , so $S_{y}(X) \geq 3$. Thus

$$
3+S(X) \leq S_{y}(X)+S(X)=S(X \cup\{y\}) \leq 9,
$$

which implies $S(X) \leq 6$. By Lemma 2, this implies $P(X) \leq 2^{3}$. By assumption, $S(X) \geq 6$, so we have $6+S_{y}(X) \leq S(X)+S_{y}(X)=S(X \cup\{y\}) \leq 9$, which implies $S_{y}(X) \leq 3$. Since every edge in $G$ has multiplicity at least 1 and $|X|=3$, we must have $w(y x)=1$ for all $x \in X$. Therefore $P(G)=P([n] \backslash X) P(X) \leq P([n] \backslash X) 2^{3}$.

Fact 1. For all $n \geq 4$ and $1 \leq i<n, \operatorname{ex}\left(n,\left\{C_{3}, C_{4}\right\}\right) \geq \operatorname{ex}\left(n-i,\left\{C_{3}, C_{4}\right\}\right)+i$.

Proof. Suppose $n \geq 4$ and $1 \leq i<n$. Fix $G=([n-i], E)$ an extremal $\left\{C_{3}, C_{4}\right\}$-free graph. Let $G^{\prime}=\left([n], E^{\prime}\right)$ where $E^{\prime}=E \cup\{n 1,(n-1) 1, \ldots,(n-i+1) 1\}$. Then $G^{\prime}$ is $\left\{C_{3}, C_{4}\right\}$-free graph because $G=G^{\prime}[n-i]$ is $\left\{C_{3}, C_{4}\right\}$-free and because the elements of $[n] \backslash[n-i]$ all have degree 1 in $G^{\prime}$. Therefore $\operatorname{ex}\left(n,\left\{C_{3}, C_{4}\right\}\right) \geq \operatorname{ex}\left(n-i,\left\{C_{3}, C_{4}\right\}\right)+\left|E^{\prime} \backslash E\right|=\operatorname{ex}\left(n-i,\left\{C_{3}, C_{4}\right\}\right)+i$.

We now prove Theorem 13. We will use that $\operatorname{ex}\left(4,\left\{C_{3}, C_{4}\right\}\right)=3$, $\operatorname{ex}\left(5,\left\{C_{3}, C_{4}\right\}\right)=5$, and $\operatorname{ex}\left(6,\left\{C_{3}, C_{4}\right\}\right)=6($ see $[10])$.

Theorem 13. For all $n \geq 4$ and $G \in F_{\leq 2}(n, 4,9), P(G) \leq 2^{\operatorname{ex}\left(n,\left\{C_{3}, C_{4}\right\}\right)}$. 
Proof. We proceed by induction on $n$. Assume first $4 \leq n \leq 6$ and $G \in F_{\leq 2}(n, 4,9)$. If $P(G)=0$ then we are done. If $G \in D(n)$, then we are done by Lemma 11. So assume $P(G)>0$ and $G \in F_{\leq 2}(n, 4,9) \backslash D(n)$. By definition of $D(n)$ this means $G \notin F(n, 3,5)$, so there is $X \in\left(\begin{array}{c}{[n]} \\ 3\end{array}\right)$ such that $S(X) \geq 6$. By Lemma [12, this implies $P(G) \leq P([n] \backslash X) 2^{3} \leq 2^{\left(\begin{array}{c}n-3 \\ 2\end{array}\right)+3}$, where the second inequality is because $\mu(G) \leq 2$. The explicit values for $\operatorname{ex}\left(n,\left\{C_{3}, C_{4}\right\}\right)$ show that for $n \in\{4,5,6\}$, $2^{\left(\begin{array}{c}n-3 \\ 2\end{array}\right)+3} \leq 2^{\operatorname{ex}\left(n,\left\{C_{3}, C_{4}\right\}\right)}$. Consequently, $P(G) \leq 2^{\left(\begin{array}{c}n-3 \\ 2\end{array}\right)+3} \leq 2^{\operatorname{ex}\left(n,\left\{C_{3}, C_{4}\right\}\right)}$.

Suppose now $n \geq 7$ and assume by induction that for all $4 \leq n^{\prime}<n$ and $G^{\prime} \in F_{\leq 2}\left(n^{\prime}, 4,9\right)$, $P\left(G^{\prime}\right) \leq 2^{\operatorname{ex}\left(n^{\prime}, 4,9\right)}$. Fix $G \in F_{\leq 2}(n, 4,9)$. If $P(G)=0$ then we are done. If $G \in D(n)$, then we are done by Lemma 11, So assume $P(G)>0$ and $G \in F_{\leq 2}(n, 4,9) \backslash D(n)$. By definition of $D(n)$ this means $G \notin F(n, 3,5)$, so there is $X \in\left(\begin{array}{c}{[n]} \\ 3\end{array}\right)$ such that $S(X) \geq 6$. By Lemma 12, this implies $P(G) \leq P([n] \backslash X) 2^{3}$. Clearly there is $H \in F_{\leq 2}(n-3,4,9)$ such that $G[[n] \backslash X] \cong H$. By our induction hypothesis applied to $H, P([n] \backslash X)=P(H) \leq 2^{\operatorname{ex}\left(n-3,\left\{C_{3}, C_{4}\right\}\right)}$. Therefore

$$
P(G) \leq P([n] \backslash X) 2^{3} \leq 2^{\operatorname{ex}\left(n-3,\left\{C_{3}, C_{4}\right\}\right)+3} \leq 2^{\operatorname{ex}\left(n,\left\{C_{3}, C_{4}\right\}\right)},
$$

where the last inequality is by Fact 1 with $i=3$.

We will use the following lemma to prove Theorem 14, Observe for all $n \geq 2, \operatorname{ex}_{\Pi}(n, 4,9)>0$ implies that for all $G \in \mathcal{P}(n, 4,9)$, every edge in $G$ has multiplicity at least 1 . We will write $x y z$ to denote the three element set $\{x, y, z\}$.

Lemma 13. Suppose $n \geq 4$ and $G=([n], w) \in \mathcal{P}(n, 4,9)$ satisfies $\mu(G) \geq 3$. Then one of the following hold.

(i) There is $x y z \in\left(\begin{array}{c}{[n]} \\ 3\end{array}\right)$ such that $\mu(G[[n] \backslash x y z]) \leq 2$ and $P(G) \leq 6 \cdot P([n] \backslash x y z)$.

(ii) There is $x y \in\left(\begin{array}{c}{[n]} \\ 2\end{array}\right)$ such that $\mu(G[[n] \backslash x y]) \leq 2$ and $P(G) \leq 3 \cdot P([n] \backslash x y)$.

Proof. Suppose $n \geq 4$ and $G=([n], w) \in \mathcal{P}(n, 4,9)$ is such that $\mu(G) \geq 3$. Fix $x y \in\left(\begin{array}{c}{[n]} \\ 2\end{array}\right)$ such that $w(x y)=\mu(G)$. We begin by proving some preliminaries about $G$ and $x y$. We first show $w(x y)=3$. By assumption, $w(x y) \geq 3$. Suppose towards a contradiction $w(x y) \geq 4$. Choose some $u \neq v \in[n] \backslash x y$. Since every edge in $G$ has multiplicity at least $1,5+w(x y) \leq S(\{x, y, u, v\}) \leq 9$. This implies $w(x y) \leq 9-5=4$, and consequently $w(x y)=4$. Combining this with the fact that every edge has multiplicity at least 1 , we have

$$
9 \leq 4+w(u v)+w(u x)+w(v x)+w(y u)+w(y v)=S(\{x, y, u, v\}) \leq 9 .
$$

Consequently, $w(u v)=w(u x)=w(v x)=w(y u)=w(y v)=1$. Since this holds for all pairs $u v \in\left(\begin{array}{c}{[n]} \\ 2\end{array}\right) \backslash x y$, we have shown $P(G)=w(x y)=4$. Because $n \geq 4$, Fact 1 implies

$$
2^{\operatorname{ex}\left(n,\left\{C_{3}, C_{4}\right\}\right)} \geq 2^{\operatorname{ex}\left(4,\left\{C_{3}, C_{4}\right\}\right)}=2^{3}>4=P(G) .
$$

Combining this with Lemma 10 shows $P(G)<2^{\operatorname{ex}\left(n,\left\{C_{3}, C_{4}\right\}\right)} \leq \operatorname{ex}_{\Pi}(n, 4,5)$, a contradiction. Thus $\mu(G)=w(x y)=3$. We now show that for all $u v \in\left(\begin{array}{c}{[n]} \\ 2\end{array}\right) \backslash x y, w(u v) \leq 2$. Fix $u v \in\left(\begin{array}{c}{[n]} \\ 2\end{array}\right) \backslash x y$ and suppose towards a contradiction $w(u v) \geq 3$. Choose some $X \in\left(\begin{array}{c}{[n]} \\ 4\end{array}\right)$ containing $\{x, y, u, v\}$. Because every edge in $G$ has multiplicity at least 1 , we have that $S(X) \geq w(u v)+w(x y)+4 \geq 10$, a contradiction. Thus $w(u v) \leq 2$ for all $u v \in\left(\begin{array}{c}{[n]} \\ 2\end{array}\right) \backslash x y$. We now show that for all $z \in[n] \backslash x y$, at most one of $w(x z)$ or $w(y z)$ is equal to 2. Suppose towards a contradiction there is $z \in[n] \backslash x y$ such that $w(z x)=w(z y)=2$. Note $S(x y z) \geq 7$. So for each $z^{\prime} \in[n] \backslash x y z, S_{z^{\prime}}(x y z) \leq 9-S(x y z)=9-7=2$. 
But since every edge has multiplicity at least 1 this is impossible. Thus for all $z \in[n] \backslash x y$, at most one of $w(x z)$ or $w(y z)$ is equal to 2 .

We now prove either (i) or (ii) holds. Suppose there is $z \in[n] \backslash x y$ such that one of $w(z x)$ or $w(z y)$ is equal to 2 . Then by what we have shown, $\{w(x y), w(z x), w(z y)\}=\{3,1,2\}$, and consequently $P(x y z)=6$. By Lemma 12, since $S(x y z) \geq 6$, we have that

$$
P(G)=P(x y z) P([n] \backslash x y z)=6 \cdot P([n] \backslash x y z) .
$$

By the preceding arguments, $\mu(G[[n] \backslash x y z])) \leq 2$. Thus (i) holds. Suppose now that for all $z \in[n] \backslash x y, w(x z)=w(y z)=1$. Then $P(G)=w(x y) P([n] \backslash x y)=3 \cdot P([n] \backslash x y)$. By the preceding arguments, $\mu(G[[n] \backslash x y]) \leq 2$. Thus (ii) holds.

Theorem 14. For all $n \geq 4, \mathcal{P}(n, 4,9) \subseteq F_{\leq 2}(n, 4,9)$.

Proof. Fix $n \geq 4$ and $G=([n], w) \in \mathcal{P}(n, 4,9)$. Suppose towards a contradiction $G \notin F_{\leq 2}(n, 4,9)$. We show $P(G)<2^{\operatorname{ex}\left(n,\left\{C_{3}, C_{4}\right\}\right)}$, contradicting that $G$ is product-extremal (since by Lemma 10 , $\left.2^{\operatorname{ex}\left(n,\left\{C_{3}, C_{4}\right\}\right)} \leq \operatorname{ex}_{\Pi}(n, 4,9)\right)$.

Since $G \notin F_{\leq 2}(n, 4,9)$, either (i) or (ii) of Lemma 13 holds. If (i) holds, choose $x y z \in\left(\begin{array}{c}{[n]} \\ 3\end{array}\right)$ with $\mu(G[[n] \backslash x y z]) \leq 2$ and $P(G) \leq 6 \cdot P([n] \backslash x y z)$. Let $H \in F_{\leq 2}(n-2,4,9)$ be such that $G[[n] \backslash x y] \cong H$. If $n \in\{4,5,6\}$, then $P(G) \leq 6 \cdot P(H) \leq 6 \cdot 2^{\left(\begin{array}{c}n-3 \\ 2\end{array}\right)}<2^{\operatorname{ex}\left(n,\left\{C_{3}, C_{4}\right\}\right)}$, where the second inequality is because $\mu(H) \leq 2$, and the strict inequality is from the exact values for $\operatorname{ex}\left(n,\left\{C_{3}, C_{4}\right\}\right)$ for $n \in\{4,5,6\}$. If $n \geq 7$, then by Lemma 13 and because $n-3 \geq 4, P(H) \leq 2^{\operatorname{ex}\left(n-3,\left\{C_{3}, C_{4}\right\}\right)}$. Therefore,

$$
P(G) \leq 6 \cdot P(H) \leq 6 \cdot 2^{\operatorname{ex}\left(n-3,\left\{C_{3}, C_{4}\right\}\right)}<2^{\operatorname{ex}\left(n-3,\left\{C_{3}, C_{4}\right\}\right)+3} \leq 2^{\operatorname{ex}\left(n,\left\{C_{3}, C_{4}\right\}\right)},
$$

where the last inequality is by Fact 1. If (ii) holds, choose $x y \in\left(\begin{array}{c}{[n]} \\ 2\end{array}\right)$ with $\left.\mu(G[[n] \backslash x y])\right) \leq 2$ and $P(G) \leq 3 \cdot P([n] \backslash x y)$. Let $H \in F_{\leq 2}(n-2,4,9)$ be such that $G[[n] \backslash x y] \cong H$. If $n \in\{4,5\}$, then $P(G) \leq 3 \cdot P(H) \leq 3 \cdot 2^{\left(\begin{array}{c}n-2 \\ 2\end{array}\right)}<2^{\operatorname{ex}\left(n,\left\{C_{3}, C_{4}\right\}\right)}$, where the second inequality is because $\mu(H) \leq 2$, and the strict inequality is from the exact values for $\operatorname{ex}\left(n,\left\{C_{3}, C_{4}\right\}\right)$ for $n \in\{4,5\}$. If $n \geq 6$, then $n-2 \geq 4$ and Lemma 13 imply $P(H) \leq 2^{\operatorname{ex}\left(n-2,\left\{C_{3}, C_{4}\right\}\right)}$. Therefore,

$$
P(G) \leq 3 \cdot P([n] \backslash x y) \leq 3 \cdot 2^{\operatorname{ex}\left(n-2,\left\{C_{3}, C_{4}\right\}\right)}<2^{\operatorname{ex}\left(n-2,\left\{C_{3}, C_{4}\right\}\right)+2} \leq 2^{\operatorname{ex}\left(n,\left\{C_{3}, C_{4}\right\}\right)},
$$

where the last inequality is by Fact 1 .

Proof of Theorem 5. Fix $n \geq 4$ and $G \in \mathcal{P}(n, 4,9)$. By Theorem 14, $G \in F_{\leq 2}(n, 4,9)$. By Theorem 13, this implies $P(G) \leq 2^{\operatorname{ex}\left(n,\left\{C_{3}, C_{4}\right\}\right)}$. By Lemma10, $P(G) \geq 2^{\operatorname{ex}\left(n,\left\{C_{3}, C_{4}\right\}\right)}$. Consequently, $P(G)=2^{\operatorname{ex}\left(n,\left\{C_{3}, C_{4}\right\}\right)}=\operatorname{ex}_{\Pi}(n, 4,9)$.

\section{References}

[1] József Balogh, Robert Morris, and Wojciech Samotij, Independent sets in hypergraphs, J. Amer. Math. Soc. 28 (2015), no. 3, 669-709. MR 3327533

[2] J. A. Bondy and Zs. Tuza, A weighted generalization of Turán's theorem, J. Graph Theory 25 (1997), no. 4, 267-275. MR 1459892 (99c:05097)

[3] G. Dirac, Extensions of turán's theorem on graphs, Acta Mathematica Academiae Scientiarum Hungarica 14 (1963), no. 3, 417-422. 
[4] P. Erdős, Some recent results on extremal problems in graph theory. Results, Theory of Graphs (Internat. Sympos., Rome, 1966), Gordon and Breach, New York; Dunod, Paris, 1967, pp. 117123 (English); pp. 124-130 (French). MR 0227049

[5] _ On some new inequalities concerning extremal properties of graphs, Theory of Graphs (Proc. Colloq., Tihany, 1966), Academic Press, New York, 1968, pp. 77-81. MR 0232703

[6] Paul Erdős, Daniel J. Kleitman, and Bruce L. Rothschild, Asymptotic enumeration of $K_{n}$-free graphs, Colloquio Internazionale sulle Teorie Combinatorie (Rome, 1973), Tomo II, Accad. Naz. Lincei, Rome, 1976, pp. 19-27. Atti dei Convegni Lincei, No. 17. MR 0463020 (57 \#2984)

[7] V. Falgas-Ravry, O'Connell K., Strmberg J., and Uzzell A., Multicolour containers and the entropy of decorated graph limits, arXiv:1607.08152 [math.CO], 2016.

[8] Zoltán Füredi and André Kündgen, Turán problems for integer-weighted graphs, J. Graph Theory 40 (2002), no. 4, 195-225. MR 1913847 (2003g:05075)

[9] — Turán problems for integer-weighted graphs, J. Graph Theory 40 (2002), no. 4, 195225. MR 1913847 (2003g:05075)

[10] David K. Garnick, Y. H. Harris Kwong, and Felix Lazebnik, Extremal graphs without threecycles or four-cycles, Journal of Graph Theory 17 (1993), no. 5, 633-645.

[11] R. L. Graham, M. Grötschel, and L. Lovász (eds.), Handbook of combinatorics. Vol. 1, 2, Elsevier Science B.V., Amsterdam; MIT Press, Cambridge, MA, 1995. MR 1373655

[12] Daniel J. Kleitman and Kenneth J. Winston, On the number of graphs without 4-cycles, Discrete Math. 41 (1982), no. 2, 167-172. MR 676877

[13] J.A. Kuchenbrod, Extremal problems on weighted graphs, Ph.D. thesis, University of Kentucky, 1999.

[14] D. Mubayi and C. Terry, An extremal graph problem with a transcendental solution, arXiv:1607.07742, 2016.

[15] David Saxton and Andrew Thomason, Hypergraph containers, Inventiones mathematicae 201 (2015), no. 3, 925-992 (English).

[16] M. Simonovits, A method for solving extremal problems in graph theory, stability problems, Theory of Graphs (Proc. Colloq., Tihany, 1966), Academic Press, New York, 1968, pp. 279319. MR 0233735 